\title{
NOTIONS DE GRAMMAIRE LO-LO
}

\author{
DIALECTE $A-H I$
}

PAR

\section{ALFRED LIETARD}

de la Société des Missions étrangères de Paris.

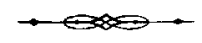

\section{I. - Les Sons et les Mots.}

1. - Voyelles. - Les voyelles du dialecte A-hi sont: $a, e$, $\ell, \grave{e}, \hat{e}, o, e u, o u$.

$e$ est bref; très-légèrement senti: eu est long, bien senti, sans exagération cependant.

$e u$ correspond à $o^{\prime}, o u$ correspond à $u$ des transcriptions scientifiques.

$u$ français n'existe pas chez les A-hi; par contre il existe fort bien dans certains dialectes du Seu-tch'ouan 四川 et du Koui-tcheou 貴州.

Les trois royelles $a, i$ et $o$ sont tantôt brèves, tantôt longues. Dans le second cas, on les marquera du signe ordinaire de la longue; dans le premier, elle ne porteront ancien signe diacritique. Ex.:

$$
\begin{array}{ll}
b a^{4}, \text { s'amuser } & b \bar{a}, \text { bifurquer } \\
c h a^{1}, \text { or } & c h \bar{a}^{1}, \text { déchirer } \\
p i^{1}, \text { mouvoir } & p^{\prime} \bar{\imath} \text { nuire } \\
p^{\prime} i^{1}, \text { acconpler } & p \bar{\imath}, \text { sacrifier } \\
d z 0^{4}, \text { manger } & p \bar{a}-d z \bar{a}, \text { natte } \\
c h o^{1}, \text { ail } & c h \bar{o}, \text { chercher }
\end{array}
$$

Plusieurs mots terminés en o se prononcent avec, avant l'o, un ou très-légèrement senti. Dans le Dictionnaire, on signalera ces mots en écrivant, par ex.: $s \bar{o}^{4}$, sou $0^{4}$, vivre. 
2. - Tons. - Les voyelles peuvent être affectées de quatre tons, correspondant au $1 \mathrm{er}$, au $2 \mathrm{e}$, au $3 \mathrm{e}$ et au $4 \mathrm{e}$ ton chinois. Je les distinguerai par des chiffres (de 1 à 4 ) placés un peu en haut et à droite des mots.

le 1er ton (上平聲 cháng p'în chên des Chinois) se présente sous deux formes: le ton égal supérieur ordinaire des Chinois; et le ton égal supérieur plus haut de deux notes (musicales) que le ton égal supérieur ordinaire des Chinois (voix presque criarde). Les mots affectés de ce ton ne porteront aucun chiffre. Ex.:

$$
t \bar{a}-m i^{1} \text {, rizière } \quad b i^{4}-t a^{2}-m o^{3} \text {, grand }
$$

En lo-lo, quand les mots entrent en composition, ou même suivant leur simple position dans la phrase, ils changent facilement de ton. Partout cependant je note les mêmes mots aux mêmes tons pour éviter la confusion.

3. - Des mots terminés normalement en eu changent parfois cette voyelle en ou, en $a$; ou en $\grave{e}, \hat{e}, a$. Ex.:

$b e u^{3}$, avoir, se dira aussi $b o u^{3}$ ou $b a^{3}$;

ngeu, être $, \quad, n g \hat{e}^{3}$ ou $n g a^{3}$.

4. - La confusion des finales $o$ et surtout $e u$ avec ou est encore plus fréquente. Ex.:

$$
\begin{aligned}
& a^{1}-n \bar{o}^{1} \text {, singe, } \quad \text { se dira aussi } a^{1}-n o u^{1} \text {; } \\
& \text { cho }{ }^{1} \text {, ail, }, \text { " chou }{ }^{1} \text {; } \\
& \text { cheu }{ }^{3}-m o^{3} \text {, cadavre, n } n \quad \text { cheu } u^{3}-m e u^{3} \text {; } \\
& \text { meu, enseigner, } \quad, \quad \text { mou. }
\end{aligned}
$$

5. - Semi-voyelles. - Les semi-voyelles sont: $y$ et $w$. $Y$ peut-être soit médian (ex.: by $e^{3}$, dire; ly $e^{2}$, main; $n y i^{3}$, s'asseoir), soit initial (ex.: $y a^{3}$, oui; $y e^{4}$, poule; $y i^{3}$, eau). $W$ est initial (ex.: $w o^{2}$, aiguille) ou médian dans les mots empruntés au chinois (ex.: $k w a^{2}$, administrer).

Rem. - Certains mots termiués en $o$, en ou prennent souvent une finale adventice $a$; et ccrtains autres terminés en $e u$, en ou, une finale adventice $\dot{e}$. Ainsi $n g \bar{o}^{1}$, falloir, ou $n g o u^{1}$ deviennent souvent

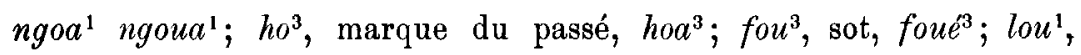
raccommoder, louél. Les finales adventices ont pour effet de transformer la voyelle précédente en semi-voyelle (ngwa, hwa, fwé, lwé); mais ce serait rendre le mot méconnaissable que de noter cette 
semi-voyelle par une lettre spéciale dans la transcription. C'est pourquoi, soit initiul, soit médian, $w$ sera rendu partout par ou. Ex.: ouo ${ }^{2}$, aiguille, pour wo; etc.

6. - Consonnes. - Les consonnes sont: $b, c h, d, d j, d z, f$, $g$ (toujours dur), $h, j, k, l, m, n, n g$, (nasale gutturale), $p, r, s, t$, $t c h, t s, v, z$.

Il importe de ne pas confondre $j$ et $z$, qui ont à peu près la même valeur qu'en français (ex.: $j o^{3}$, prendre; $z 0^{4}$, fils), avec $d j$ et $d z$, où le $d$ doit être senti fortement (ex.: $d j 0^{4}$, aimer; $d z 0^{4}$, manger).

7. - L'aspiration initiale est toujours marquée par $h$. Devant $a, \dot{e}, \grave{e}, \hat{e}, e u, o, o u$, l'aspiration est très-douce, et parfois même difficile à distinguer d'un $r$ frôlé. Devant $i$ et $y$ et devant la consonne $l$, elle se rapproche au contraire de la sifflante palatale, marquée dans les transcriptions scientifiques par $\$$ (ex.: $A^{1}-h i^{1} ; h i^{3}-p i^{4}$, puce; $h l o^{3}-b o^{3}$, lune; prononcez $A^{1} \cdot c h i^{1}$ ou $A^{1}-s h i^{1} ; c h i^{3}-p i^{4}$ ou $s h i^{3}-$ $p i^{4} ; c h l o^{3}-b o^{3}$ ou $\left.s h l o^{3}-b o^{3}\right)$.

L'aspiration ne se trouve à l'intérieur des mots qu' après les consonnes $k, p, t, t c h$, et $t s$. Elle est très-fortement sentie, comme en chinois. Je la marque par une apostrophe. Ex.: $k{ }^{\prime} \bar{a}-n o^{3}$, combien.

Enfin il existe trois espèces de mots que j’écris ' $e,{ }^{c} e u,{ }^{c} i$, où l'émission de la voyelle est accompagnée d'un souffle fortement accentué. Ex.: ' $i^{2}$, huit; ${ }^{c} e u^{3}$, appeler. Cette espèce de mots, assez rares en A-hi, sont au contraire très-communs dans certains dialectes.

Rem. I. - Dans les mots $k i$ et $k y e ́$, le $k$ initial est souvent prononcé comme th. Ex.: $k i^{3}$, soleil, ou $t h i^{3}$, (prononcez $t c h i^{3}, t s h i^{3}$ ); kyé, corde ou thyé. (prononcez tchyé, tshyé).

Rem. 2. - De même, dii, se change fort souvent en dyi. Ex.: $d j i^{4}-m o^{4}$, bête ou $d y i^{4}-m o^{4}$; lou ${ }^{4}-d j i^{4}$, Chinois, ou lou $u^{4} d y i^{4}$.

Rem. 3. - Les mots en ro sont souvent confondus avec ceux en ouo (wo). Ex.: $r o^{4}-d o^{3}$, neiger ou $o u o^{4}-d o^{3}$.

Rem. 4. - Certains dialectes affectent des consonnes initiales redoubléés. Ex.: $f f{ }^{1}$, vêtir; $d d i^{2}$, il suffit.

8. - Dans quelques mots a-hi commençant par $m$, la voyelle finale tombe et l'm prend la valeur d'une sonante. Ex.:

$a^{1}-p e u-m^{\prime 4}$, vieillard, et $a^{1}-p^{\prime} i^{4}-m^{\prime 4}$, vieille femme, pour 
$a^{1}-p e u-m o u^{4}$ et $a^{1}-p^{1} i^{4}-m o u^{4}$, qui s'emploient également;

$a^{1}-l e u-m^{\prime 4}$, ou $a^{1}-l o u-m^{\prime 4}$, cheval, ou $m^{\prime 4}$ equivaut à $m o^{4}$, cheval. $a^{1}-l a^{3}-l i-m^{4}$, âne, pour $a^{1}-l a^{3}-l i-m o^{4}$.

$m^{4}-b \bar{a}^{1}$, tirer du fusil, pour mout $-b a^{1}$.

9. - En a-hi, et dans toutes les tribus lo-lo du Yun-nan que je connais, les consonnes ne peuvent être qu' initiales, jamais finales. Les mots lo-lo, tous rigoureusement monosyllabiques, sont donc composés, ou d'une simple voyelle (ex.: $a^{4}$, non), ou, ce qui est le cas général, d'une consonne suivie d'une voyelle (ex.: $m o^{4}$, cheval; $t s^{\prime} \alpha^{3}$, graisse; $k^{\prime} y e^{3}$, village; lou ${ }^{1}$, paître; $t c h a^{2}$, bon).

10. - Toutefois, si les mots lo-lo sont d'un monosyllabisme rigoureux, il n'en faut pas moins dire que nombre de substantifs, quelques adjectifs et verbes sont composés de plusieurs monosyllabes que l'usage associe étroitement. D'une façon générale, on peut affirmer que chacun de ces monosyllabes a un sens par lui-même: en plusieurs cas, nous avons pu découvrir ce sens nous-même, mais. dans beaucoup d'autres notre analyse n'a pas encore réussi à la pénétrer. Enfin il semble bien que certains de ces mots soient de simples particules, vides, actuellement du moins, de toute signification. Nous en donnerons quelques exemples empruntés à chaque catégorie.

11. - $1^{\circ}$ Substantifs. - $a$ ) Dans des substantifs composés

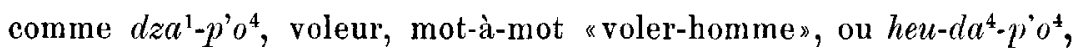
forgeron, mot-à-mot «fer-battre-homme», les mots composants gardent leur sens propre et se laissent facilement identifier.

b) Dans d'autres substantifs composés, $o^{1}-k \bar{o}$, tête, $d j i^{4}-m o^{4}$, bête, ly $e^{2}-p e u^{1}$, main, $n i^{1}-m o^{3}$, cœur, il semble bien que les mots $o^{1}, d j i^{4}$, $l y e^{2}, n i^{1}$ à eux seuls désignent la tête, l'animal, la main, le cœur: en effet on les retrouve avec ce sens dans nombre d'autres composés (ex.: $o^{1}-t i^{3}$, turban; $o^{1}-t^{\prime} \bar{o}^{3}$, calotte; ly $e^{2}-s e u^{4}$, ongle; $l y e^{2}-t c h o^{3}$, bracelet; $n i^{1}-k^{\prime} y e^{2}$, méchant), et l'on dira aussi bien $d j i^{4}-l o u^{1}$ que $d j i^{4}-$ $m o^{4}$ lou ${ }^{1}$, faire paître les bêtes. - Mais je ne saurais être aussi affirmatif pour le second élément de ces quatre mots que je n'ai pu isoler jusqu'ici. Et remarquez que, pour le mot $o^{1}-k \bar{o}$ par exemple, on ne dira jamais $o^{1} n o^{3}$, avoir mal à la tête, mais toujours $o^{1}-k \bar{o} n o^{3}$.

c) A mon avis, dans $a^{1}-b a^{4}$, père; $a^{1}-m o^{3}$, mère; $i^{1}-2 o^{4}$, fils ; $a^{1}-p o u$, 
grand-père; $a^{1}$-beu ${ }^{3}$, tubercule; $i^{1}-s \hat{e}^{3}$, esprit; $i^{1}$-s $\hat{e}^{2}$, fumée, l'élément initial, $a^{1}$ ou $i^{1}$, est une simple particule n'ayant aucun sens. Ce qui me le fait croire, c'est que l'a se change souvent en $i^{1}$ et qu'on dit aussi bien $i^{1}-b a^{2} ; i^{1}-m o^{3} ; i^{1}-b e u^{3}$ que $a^{1}-b a^{4} ; a^{1}-m o^{3} ; a^{1}-b e u^{3}$. De plus, ees particules sont souvent supprimées, et l'on dira, par exemple: Cheu-do $k e u^{1} b a^{4}$, le père de Cheu-do; Cheu-do keu $m o^{3}$, la mère de Cheu-do; Cheu-do keu ${ }^{1}$ pou, le grand-père de Cheu-do; $m i^{1} s \hat{e}^{3}$, esprit de la terre; mou ${ }^{1}$-teu $s \grave{e}^{2}$, fumée.

12. - $2^{\circ}$ Adjectifs. - a) Nous ne faisons que mentionner ici les adjectifs formés d'un seul mot auquel on ajoute la particule $m o^{3}$, signe particulier de l'adjectif dans le dialecte a-hi. Ex.:

tcha $\alpha^{2}$, bon: $t s^{\prime} o u^{3} t c h a^{2}$, ou $t s^{\prime} o u^{3} t c h a^{2}-m o^{3}$, homme bon. Che $u^{3}$, mourir: $t s^{\prime} o u^{3} c h e u^{3}$, ou $t s^{\prime} o u^{3} c h e u^{3}-m o^{3}$, homme mort.

b) Dans des adjectifs comme $u i^{1}-k^{\prime} y e^{2}$, méchant, mot-à-mot «cœur mauvais», les deux éléments sont aisément reconnaissables. Et l'on dira indifféremment: $t s^{\prime} o u^{3} k^{\prime} y e^{2} ; t s^{\prime} o u^{3} k^{\prime} y e^{2}-m n o^{3} ; t s^{\prime} o u^{3} n i^{1}-k k^{\prime} y e^{2}$; $t s^{\prime} o u^{3} n i^{1}-k^{\prime} y e^{2}-m o^{3}$; homme méchant.

c) Il y a parmi les adjectifs des composés beaucoup plus complexes encore. Prenons par exemple $a^{1}-t^{1} o^{3}-m o^{3}-y e^{3}$, blanc, ou $a^{1}-n y e^{4}-$ $m o^{3}-y e^{3}$, noir. L' $a^{1}$ initial et le $y e^{3}$ final des deux mots paraissent bien être deux particules dépourvues de sens, mais qui affectent respectivement ces places. $M o^{3}$ est le signe particulier de l'adjectif. Remarquons enfin que $t^{\prime} o^{3}$ et $n y \dot{e}^{4}$ à eux seuls signifient blanc et noir. On dira indistinctement pour étoffe blanche, $p^{\prime} o^{3} t^{\prime} o^{3} ; p^{\prime} o^{3} t^{\prime} o^{3}-$ $m o^{3} ; p^{\prime} o^{3} \quad a^{1}-t^{\prime} o^{3} ; p^{\prime} o^{3}-t^{\prime} o^{3}-m o^{3}-y e^{3} ; p^{\prime} o^{3} \quad a^{1}-t^{\prime} o^{3}-m o^{3} ; p^{\prime} o^{3} a^{1}-t^{\prime} o^{3}-m o^{3}-$ $y e^{3}$; et il en est de même pour $n y \hat{e}^{4}$, noir.

13. - $3^{\circ}$ Verbes. - Les verbes composés sont plus rares. Examinons-en quelques-uns; comme, par ex.:

$N i^{3}$-dzêt $\hat{e}^{4}$ monter (à cheval); composé de $n i^{3}$, s'asseoir, et $d z \hat{e}^{4}$, enfourcher.

$G \bar{o}^{3}-f f{ }^{1}$, revêtir; gou ${ }^{3}-y i^{2}$, se coucher; composés tous deux de $g \bar{o}^{3}$ ou gou $u^{3}$, faire; et de $f f^{2}$, vêtir; et $y i^{2}$, se coucher.

Dou $u^{4}-k^{\prime} o u^{2}$, ou dou $-k^{\prime} o u^{2}-b y e^{3}$, répondre; composé de $d o u^{4}$, paroles, $k^{\prime} o u^{2}$, restituer, rendre, et $b y e^{3}$, dire. Ex.: $d o u^{4}-k^{\prime} o u^{2} n g \bar{o}^{1}$, on $d o u^{4}$ $k^{\prime} o u^{2} b y e^{3} n g \bar{o}^{1}$, il faut répondre. Le sens de rendre que nous attribuons à $k^{\prime} u^{2}$ est confirmé par le composé suivant. 
$D o^{4}-k^{\prime} o u^{2}$, restituer. Ex.: $S o^{3} m \hat{e}^{3} d o^{4}-k^{\prime} o u^{2} n g \bar{o}^{1}$, il faut restituer la réputation du prochain, m. à. m., [d'] autrui [le] nom restituer falloir. Du reste $k$ 'ou à lui seul signifie restituer, et pour demander: comment faut-il la restituer (cette réputation)?, on dira fort bien: $k^{\prime} \bar{a}-z e u^{4} k^{\prime} u^{2} n g \bar{o}^{1}$ ?, m. à m. comment restituer falloir. Mais j'ignore la signification de $d o^{4}$ dans $d o^{4} \cdot k^{\prime} o u^{2}$.

\section{II. - De l'Article.}

14. - Il n'y a en a-hi ni article défini ni article indéfini: on exprime le substantif sans addition. Ainsi, suivant le contexte, he ${ }^{3}$ signifie la (ou) une maison; $t s^{\prime} o u^{3}, \mathrm{l}^{\prime}(\mathrm{ou})$ un homme; go $\mathrm{o}^{4}$ le (ou) du sarrasin; $y i^{3}-d y e^{4}$, l' (ou) de l'eau; $k a^{4}-b i^{4}$, les (ou) des habits.

15. - Toutefois un, jouant le rôle d'article indéfini, est souvent exprimé. Ex.: $t s^{\prime} o u^{3} t^{\prime} i^{4}-l e u^{4}$, ou simplement $t s^{\prime} o u^{3}$, un homme; $k^{\prime} y^{4} k^{\prime} y e^{2} t^{\prime} i^{4}-l e u^{4}$, ou simplement $k^{\prime} i^{4} k^{\prime} y e^{2}$, un chien méchant.

\section{III. - Substantifs.}

16. - Les noms abstraits sont inconnus en lolo. Ainsi il n'y a aucun mot pour exprimer la bonté de l'homme: on rendra l'idée en disant $t s^{\prime} o u^{3}$-tcha', homme bon.

17. - Les substantifs relatifs au pays, à la religion, à la profession, etc., se forment, en A-hi, en ajoutant au substantif, à l'adjectif ou verbe, indiquant ces choses, l'un des mots; $p^{\prime} o^{4}$, individu, homme, signe du masculin; tche $u^{3}$, esclave, servir; $t s^{\prime} \circ u^{3}$, homme en général. Ex.:

$D j i^{4}-d o-h e u^{4} t s^{\prime} o u^{3}$, homme de $\mathrm{Dji}^{4}$-do-heu ${ }^{4}{ }^{1}$ )

$M o u^{4} s a^{4}-p^{\prime} o^{4}, t s^{\prime} o u^{3}$, chrétien, m. à m. (du) Ciel (du) Seigneur homme.

$N e u^{4} m o^{3} t s^{\prime} o u^{3}$, mercenuire, m. à m. travail faire homme.

$A^{1}$-leu-m'4 $m^{\prime 4}$ che ${ }^{3}$, palefrenier, m. à m. (du) cheval esclave.

$K^{\prime} i^{3}-n \bar{o}^{3} d j e u^{2}-p^{\prime} o^{4}$, cordonnier, m. à m. souliers coudre homme. $T s o^{3} m^{3} p^{\prime} o^{4}$, cuisinier, m. à m. riz (= nourriture) faire homme. Heı $d a^{4} p^{\prime} o^{4}$, forgeron, m. à m. fer battre homme.

1) Nom lo-lo dı village appelé en chinois Lan-gni-tsin 姫泥箩; m. à m. bêtes-se noyer-étang. 
18. - Les substantifs relatifs aux monuments, habitations, demeures, etc., sont presque tous formés par l'addition du mot hêे $\hat{e}^{3}$ demeure. Ex.:

$B e u^{1}-z 0^{4} h \hat{e}^{3}$, pagode, m. à m. idole maison.

$D z e u^{4}-m o u^{4} h \hat{e}^{3}$, prétoire, m. à m. mandarin maison.

19. - Un trés grand nombre de substantifs sont formés par l'addition de la particule $t o^{3}$ que je traduirais volontiers par instrument, chose, objet, avec un sens très-large; laquelle particule sert du reste à former le gérondif. Ex.:

$B o u^{4}-t s^{\prime} \hat{e}^{3}$ teu $u^{1}-t o^{3}$, moustiquaire, m. à m. moustiques intercepter instrument.

$D z \bar{a}^{1}-t o^{3}$, ciseau, m. a m. à-couper instrument.

$N o^{1}-b o^{2} s^{1} t o^{3}$, mouchoir, m. a m. nez essuyer instrument.

$D z o^{4}-t o^{3}$, vivres, $\mathrm{m}$. ̀̀ $\mathrm{m}$. à-manger choses.

$F f{ }^{1}-t o^{3}$, vêtements, m. à $\mathrm{m}$. à-vêtir choses.

20. - Nombre de substantifs peuvent avoir un diminutif formé par l'addition de $z o^{4}$, fils, enfant, petit. Ex.:

$m o^{4}$, cheval : $m o^{4}-z 0^{4}$, poulain ;

$y e^{4}$, poule: $y e^{4}-z 0^{4}$, poussin;

$l o u^{1}-m o^{3}$, pierre: $l o u^{1}-m o^{3}-z 0^{4}$, petite pierre, caillou.

Rem. - Pour les choses inanimées cependant, au lieu de la particule $z 0^{4}$, on se sert en général d'un adjectif. Ex.:

lou ${ }^{1}-m o^{3}$, pierre: $l o u^{1}-m o^{3} a^{1}-t s e u^{1}-y e^{3}$, petite pierre.

21. - Du genre. - Grammaticalement parlant, il n'y a pas de genre en lo-lo. Cependant, lorsqu'on veut désigner le sexe des personnes, on ajoute au substantif $p^{\prime} o^{4}$ pour le masculin et mo pour le féminin. Ainsi on dira $A^{1} \cdot h i^{1}-p^{\prime} o^{4}$, un A-hi, $A^{1}-h i^{1}-m o^{3}$, une A-hi; tche $u^{3}-p^{\prime} o^{4}$, un esclave, tcheu $u^{3}-m o^{3}$, une esclave; - ouo $o^{3}-m o u^{3}-p^{\prime} o^{4}$, le roi, $o u o^{3}-m o u^{3}-m o^{3}$, la reine. On dira indifféremment $g o^{3} A^{1}-h i^{1}-p^{1} o^{4}$ $n g e u^{3}$, je suis un A-hi, m. à m. moi A-hi bomme être, ou $g 0^{3} A^{1}-h i^{1} n g e u^{3}$.

22. - Pour les animaux, mâle se dit $i^{1}-p o^{1}\left(i^{1}-p^{\prime} o u^{3}\right.$ s'il s'agit de volatiles), et femelle se dit $i^{1}-m o^{3} . P o^{1}$ et $m o^{3}$ servent de suffixes. Ex.:

$m o^{4}-p o^{1}$, cheval; $m o^{4}-m o^{3}$, jument;

$y e^{4}-p^{\prime} o u^{3}, \operatorname{coq} ; y e^{4}-m o^{3}$, poule.

Remarque. - Le bœuf en général se dit $n i^{4}$; la vache, $n i^{4}-m o^{3}$. Iue taureau à un nom spécial lo-hê; le taureau châtré lo-beu'.

23. - Du pluriel. - Dans différents dialectes lo-lo, le pluriel 
est généralement indiqué par le contexte, et même s'il existe un signe spécial pour le pluriel, ce signe n'est employé qu' exceptionnellement. En a-hi cependant, la particule $h i^{4}$, signe distinctif du pluriel, est en usage constant; et, bien qu'elle puisse être omise sans nuire à la clarté de la phrase, les A-hi préfèrent en général l'employer. Ex.:

ts'ou ${ }^{3}-h i^{4} b y e^{3}$ (ou ts'ou bye $e^{3}$ ), des hommes disent.

$D j i^{4}-d o-h e u^{4} t s^{\prime} o u^{3}-h i^{4} n g e u^{3}$ (ou Dji $i^{4}-d o-h e u^{4} t s^{\prime} O u n g e u^{3}$ ), ce sont des hommes de Dji-do-heu (Lan gni tsin).

24. - Comme on le voit, la particule $h i^{4}$ se place toujours après le substantif. Si le substantif est suivi lui-même d'un adjectif qualificatif, la particule est plaçée après l'adjectif. Ex.:

$t s^{\prime} o u^{3} t c h a^{2} h i^{4}$, des hommes bons; - $t s^{\prime} o u^{3} n i^{1}-k e^{\prime} e^{2} \cdot m o^{3} h i^{4}$, des hommes méchants.

25. - Cette particule peut également se placer à la suite d'une énumération. Ex.:

$i^{1}-b a^{4} i^{1}-m o^{3} h i^{4}$, père et mère.

26. - La particule $h i^{4}$ rejetée à la fin de la proposition après le verbe prend le sens du pronom relatif ceux qui. Ex.:

$t s o^{3} d z o^{4} a^{4} k e u^{1} h i^{4}, k^{\prime} \bar{a}-z e u^{4} g \bar{o}^{3} n g o a^{1} ?$ - que doivent faire ceux qui ne peuvent manger?, $m$. à m. nourriture manger ne-pas pouvoir ceux-qui, comment faire falloir.

$M o u^{4} s^{4}-p^{1} o^{4} n y i^{3}, n e u^{4} m o^{3} h i^{3}, M i^{2}-s a^{1} a^{4} n \bar{o}^{1} h^{4}, k a^{4}-m i^{1}$ tsou ${ }^{1}$ $b e u^{3}$ ? - quelle faute commettent ceux qui, le Dimanche, travaillent et u'entendent pas la messe?, m. à m. (du) Ciel (du) Seigneur (le) jour, travail faire ceux-qui, messe ne-pas entendre ceux-qui, quelle faute avoir.

On dirait tout aussi bien sans nuire à la clarté: $M o u^{4} s a^{4}-p^{\prime} o^{4}$ $n y i^{3}, n e u^{4} m o^{3}, M i^{2}-s a^{1} a^{4} n \bar{o}^{1}, k a^{4}-m i^{1} t s o u^{1}$ beu $u^{3}$ ?, m. à m. (du) Ciel (du) Seigneur jour, travail faire, messe ne-pas entendre, quelle faute avoir.

27. - Il n'est pas inutile de faire remarquer qu'en a-hi la particule $h i^{4}$ a aussi le sens de chose. Ex.:

$\left.H i^{4} n g a^{4} h i^{4}, h i^{4} t c h \hat{e}^{3}-m o^{3} h i^{4}, t o u^{4}-o^{1}-t c h o u^{3}{ }^{1}\right) d i^{2} ; h i^{4} a^{1}-t s e u^{1}-$

1) Tou-tchou ${ }^{3}$ est le chinois 賭 $\mathrm{p}_{\mathrm{L}}^{\mathrm{p}}$ tou-tcheou; $o^{1}$ est une particule euphoniquesans signification propre (cf. $\$ 113$ ). 
$\left.z 0^{4}{ }^{1}\right) h i^{4}, h i^{4} a^{4} t c h e^{3} h i^{4}, a^{4} d i^{2}$. - Pour des choses sérieuses et pour des choses vraies, on peut faire serment; pour des futilités et pour des choses fausses, on ne le peut pas; m. àm.: choses grandes - marque du pluriel -, choses vraies - marque du pluriel jurer pouvoir; choses petites-très — marque du pluriel —, choses non vraies - marque du pluriel -, ne-pas pouvoir.

27. - On peut encore exprimer le pluriel par des tournures spéciales. Ex.:

$G o^{3} k^{\prime} i^{2} n i^{4} \operatorname{seu}^{3}-l e u^{4} v a^{3} h o^{3}$. - J'ai acheté des chèvres, m. à m. moi chèvres deux trois-numérale - acheter - signe du parfait. On pourrait dire plus simplement: $g o^{3} k i^{2} v a^{3} h o^{3}$.

28. - Régime du substantif. - Dans tous les dialectes lolo, le régime du substantif peut s'exprimer sans aucun signe particulier, simplement par la position des mots dans la phrase: le nom-régime se place alors avant le substantif dont il dépend. Ex.:

$G o^{3} b a^{4} a^{1}-l e u-m^{4}$, le cheval de mon père, m. àm. (de) moi (du) père (le) cheval.

Mou $s a^{4}-p^{\prime} o^{4} t c h o^{3}-m a^{3}$, la doctrine de Dieu, m. à m. (du) Ciel (du) seigneur (la) route.

29. - Néanmoins, tous les dialectes lo-lo possèdent au moins une particule spéciale indiquant le cas régime. La plus commune, à ma connaissance, est $d y i^{4}$ : elle existe, en particulier, en a-hi. i

30. - L'a-hi possède encore deux autres particules marquant le génitif, ke $u^{\mathrm{L}}$, il, lui, elle, et $v i^{3}$ : elles se placent également après le nom régime.

$1^{0} K e u^{1}$ ne s'emploie guère que pour indiquer la parenté, la descendance. Ex.: $S u^{4}-z \alpha^{1}-n a^{3} k e u^{1} b a^{4}$, le père de Suzanne, m. à m. Suzanne (d')elle (le) père; Suzanne son père.

$L o u^{3}-y i^{4}-z a^{1} k e u^{1} v i^{2}$, la sœur aînée de Lsouise, m. à m. Louise (d')elle (la) sœur-aînée.

Il serait également correct, mais moins élégant, de dire: $S u^{4}-z a^{1}$ $n a^{3} b a^{4}$; ou $L o u^{3}-y i^{4}-z a^{1} v i^{2}$.

31. $-2^{0}$ Dans les autres cas on emploie de préférence $v i^{3}$ ou $d y i^{4}$. Ex.:

1) Jointe à un mot exprimant la petitesse, cette particule diminutive $z o^{4}$ (cf. $\$ 20$ ) prend un sens superlatif. 
$M o u^{4} s a^{4}-p^{\prime} o^{4} v i^{3}$ tcho ${ }^{3}-m a^{3}, o^{4}-s e u^{3}-l e u^{4} b y e^{3} ?-\mathrm{Ou}: M o u^{4} s a^{4}-$ $p^{\prime} o^{4} d y i^{4} t c h o^{3}-m a^{3}, a^{4}-s e u^{3}-l e u^{4} b y e^{3} ?$ - Qui a prêché la religion? m. à m. (du) Ciel Seigneur du (la) voie, qui dire?. C.ulu

Il serait moins bien reçu, mais non pas absolument incorrect, d'employer $k e u^{1}$ dans cette phrase, au lieu de $v i^{3}$ ou de $d y i^{4}$; et l'on pourrait sans inconvénient, supprimer toute particule.

\section{IV. - Adjectifs.}

32. - Particules caractéristiques. - En a-hi, $m o^{3}$ est la particule caractéristique de l'adjectif. Elle peut du reste toujours être supprimée, à moins qu'on ne veuille éviter une confusion. Elle se place après l'adjectif. Ex.:

$t s^{\prime} O u^{3} t c h \alpha^{2}-m o^{3}$ ou $t s^{\prime} O u^{3} t c h a^{2}$, homme bon;

$t s^{\prime} o u^{3} r a^{4}-m o^{3}$ ou $t s^{\prime} o u^{3} r a^{4}$, homme grand;

$m i^{1} d \hat{e}^{4}$, bêcher la terre: $m i^{1} d \hat{e}^{4}-m o^{3}$, terre bêchée.

33. - Certains adjectifs prennent volontiers un $a^{1}$ initial. Ex.: $t^{\prime} o^{3}$, blanc, ou $a^{1}-t^{\prime} o^{3} ; n y e^{4}$, noir, ou $a^{1}-n y e^{4}$.

Ce préfixe peut du reste coexister avec le suffixe normal $m o^{3}$, et l'on a dans ce cas, $a^{1}-t^{\prime} o^{3}-m o^{3}$, blane, et $\cdot a^{1}-n y e^{4}-m o^{3}$, noir. Enfin ces adjectifs peuvent comporter encore un second suffixe, $y \dot{e}^{3}$, qui se place après $m o^{3}$, ce qui donne $a^{1}-t^{\prime} o^{3}-m o^{3}-y e^{3}$ et $a^{1}-n y e^{4}-m o^{3}-y e^{3}$.

34. - Certains adjectifs formés d'un seul mot redoublent ce mot auquel s'ajoute la particule $y e^{3}$ : ainsi $t^{\prime} o^{3}$, blanc, peut donner $t^{\prime} o^{3}$ $t^{\prime} 0^{3}-y e^{3}$. Cette répétition est emphatique et donne à l'adjectif plus de force. Lorsque l'adjectif est composé de deux mots, e'est le second seul qui est redoublé. Ex.:

$b i^{4}-t a^{2}$, grand, donne $b i^{4}-t a^{2}-t a^{2}-y e^{3} ;$

$b o^{3}-l i^{4}$, clair, donne $b o^{3}-l i^{4}-l i^{4}-y e^{3}$.

35. - Certains adjectifs expriment une idée d'exiguité, de petitesse etc., prennent volontiers la particule diminutive $20^{4}$ (cf. $\S 20$ et 114), qui se place après $m o^{3}\left(m o^{3}-z o^{4}\right)$, mais avant $y \dot{e}^{3}\left(z o^{4}-y e^{3}\right)$.

Rem. - Toutes les formes que nous venons d'énumérer (\$33-35) sont spéciales au dialecte a-hi.

36. - Adjectifs négatifs. - Les adjectifs négatifs se forment de trois manières: 
$1^{0}$ Quand l'adjectif est formé d'un seul mot, il suffit de le faire précéder de la particule négative $a^{4}$. Ex.:

ts'ou ${ }^{3} t c h a^{2}$, homme bon: $t s^{\prime} o u^{3} a^{4} t c h a^{2}$, homme pas bon.

$2^{0}$ Quand l'adjectif est composé de deux ou plusieurs mots, on le fait suivre du verbe être précédé de la négation. Ex.:

$a^{1}-t^{3} o^{3}-m o^{3}$, blanc: $a^{1}-t^{3} o^{3}-m o^{3} a^{4} n g e u^{3}$, pas blanc;

$b i^{4}-t a^{2}-t a^{2}-y e^{3}$, grand: $b i^{4}-t a^{2}-t a^{2} a^{4} y e^{3}$, pas grand.

37. - Adjectifs formés de verbes. - Un verbe peut fort bien devenir adjectif par la suffixation de $m o^{3}$, ou même sans changement aucun. Ex.:

$s \bar{o}^{4}$, vivre; $t s^{\prime} O u^{3} s \bar{o}^{4}$ ou $t s^{\prime} o u^{3} s \bar{o}^{4}-m o^{3}$, homme vivant.

$c h e u^{3}$, mourir; $t s^{\prime} \mathrm{ou}^{3}{ }^{3} c h e u^{3}$ ou $t s^{\prime} \mathrm{ou}^{3} c h e u^{3}-m o^{3}$, homme mort.

38. - Pasition de l'adjectif. - L'adjectif qualificatif se place régulièrement après le substantif qu'il qualifie. On dira donc ts'ou $t c h a^{2}$, homme bon, et jamais $t c h a^{2} t s^{\prime} o^{3}$.

Rem. - De nos jours ou peut parfois trouver quelques exceptions a cette règle chez les tribus lo-lo, ayant des rapports avec les Chinois. Ces exceptions ne sont que des reproductions serviles de tournures chinoises.

39. - En règle générale, l'adjectif ne peut être employé substantivement. On 'ne dira pas comme en français les bons, les méchants, mais hommes bons, hommes méchants, $t s^{\prime} o u^{3} t c h a^{2}, t s^{\prime} o u^{3} k^{\prime} y e^{2}$. On ne dira jamais à brûle-pourpoint: $s \bar{o}^{-4}-h i^{4} \quad c h e u^{3}-h i^{4} v \hat{e}^{3}-c h e u^{4}$, juger les vivants et les morts, $\mathrm{m}$. à $\mathbf{m}$. morts vivants juger, mais bion: $t s^{\prime} o u^{3} s \hat{o}^{4}-h i^{4} t s^{\prime} o u^{3} c h e u^{3}-m o^{3}-h i^{4} v \hat{e}^{3}-c h e u^{4}, \mathrm{~m}$. à m. hommes vivants hommes morts juger.

40. - Cependant, si un substantif accompagné d'un adjectif a déjà été énoncé et qu'on ait à le répéter au cours de la phrase, on peut très-bien, dans le second cas, ne répéter que l'adjectif. Ex.:

$t s^{\prime} o u^{3} t c h a^{2}-h i^{4}, t s^{\prime} o u^{3} n i^{1}-k^{\prime} y e^{2}-h i^{4} \quad b e u^{3}: t c h a^{2}-m o^{3}-h i^{4} m o u^{4}-k^{\prime} a^{3} d y e^{3}$ $d o^{2}, n i^{1}-k^{1} y e^{2}-h i^{4} m o u^{4}-k^{\prime} a^{3} d y e^{3} a^{4} d 0^{2}$, il $\mathrm{y}$ a des hommes bons et des hommes méchants; les bons peuvent aller au ciel, mais non pas les méchants, m. à m. hommes bons - marque du pluriel -, hommes mêchants - marque du pluriel - avoir: bons - marque pluriel - ciel monter pouvoir, mauvais - marque pluriel - ciel monter ne-pas pouroir. 
41. - Degrés de comparaison. - En a-hi il n'existe pas, à proprement parler, de comparatif: toutefois diverses tournures permettent de le rendre.

42. - Pour rendre le comparatif de supériorité exprimé en français par plus, on énonce d'abord l'objet qui possède la qualité à un degré plus élevé, puis celui qui la possède à ne degré moindre; on fait suivre ces deux termes des mots $n^{\prime} a^{4}-b b^{4}$, comparé a, et entin de l'adjectif exprimant la qualité. Ex.:

$P^{\prime} o^{3} t^{\prime} o^{3} p^{\prime} o^{3} n y e^{4} n^{3} a^{4}-6 o^{4}$ tsé, l'étoffe blanche est plus belle que l'étoffe noire, m. a m. étoffe blanche étoffe noire comparée-à belle. $G o^{3} n i^{3} n^{3} a^{4}-b o^{4} t s^{\prime} o n^{3}-m e u^{4} h o^{3}$, je suis plus âgé que toi, m. à m. moi toi comparé-à vieux-marque du parfait.

Rem. - $n^{3} a^{4}-b 0^{4}$ que j'écris parfois $n a^{4}-60^{4}$ parce qu'il est pro. noncé tel, contient en réalité trois mots $n 0^{3} a^{4} b o^{4}$.

43. - Pour rendre le comparatif d'infériorité exprimé en français par moins, ou énonce d'abord l'objet qui possède la qualité à un degré moindre, puis celui qui la possède à un degré plus élevé; on fait suivre ces deux termes du mot $k^{\prime} o^{1}$, semblable, et enfin de l'adjectif exprimant la qualité précédé de la négation. Ex.:

$P^{\prime} o^{3}$ nyi $p^{\prime} o^{3} n i^{1}-c h o u^{1}-m o^{3} k^{\prime} o^{1} a^{4} t s e ́$, l'étoffe rouge est moins belle que l'étofe verte; $\mathbf{m}$. à $\mathrm{m}$. étofle rouge étoffe varte semblable pas belle.

44. - Pour rendre le comparatif d'égalité exprimé on français par aussi, on énonce les deux termes à comparer, qu'on fait suivre du mot $k^{\prime} o^{1}$, semblable, et enfin de l'adjectif exprimant la qualité. Ex.:

$G o^{3} \quad h e^{3} \quad n i^{3} d y i^{4} \quad k^{3} o^{1} t c h a^{2}$, ma maison est aussi belle que la tienne; m. à m. moi maison toi de semblable belle.

45. - Pour rendre le comparatif portant sur plusieurs objets, ou le superlatif relatif, on énonce d'abord les objets à comparer, puis celui auquel on attribue la supériorité, et enfin l'adjectif. Ex.:

$G o^{3}-k i^{4} f o u^{1}-f o u^{3}, n i^{3} i^{1} \cdot b a^{4} t s^{\prime} o u^{3}-m e u^{4}$, ton père est plus vieux que nous tous; $m$. à $\mathbf{m}$. (de) nous tous, (de) toi père vieux.

$K i^{1} \quad s e u^{3}-t s^{\prime} e^{3}, k i^{1}-t^{\prime} e u^{1} b i^{4}-t a^{2}$, de ces arbres, celui-ci est le plus grand; m. à m. ces arbres, celui-ci grand.

46. - Le superlatif absolu s'exprime de diverses manières:

$1^{0}$ Par la répétition de l'adjectif. Ex.: 
tcha $a^{2}$, bon: $t c h a^{2} t c h a^{2}$, très-bon;

$t^{\prime} o^{3}$, gras: $t^{\prime} s o^{3}-t s^{\prime} o^{3}$, très-gras.

$2^{0}$ Par l'adjectif précédé de l'expression pé2-lét , très. Ex.:

$p e^{2}-l e^{\prime 2} t c h a^{2}$, très-bon; pé $e^{2}-l e^{2} t^{\prime} o^{3}$, très.gras.

Dans ce cas, on ajoute souvent encore le verbe être suivi de "T'adjectif répété. Exx.:

pé $e^{2}-l e^{2} t c h a^{2} n g e u^{3} t c h a^{2}$, très-bon; $p e^{2}-l e^{2} t^{\prime} s o^{3} n g e u^{3} t s^{\prime} v^{3}$, très-gras.

$30 \mathrm{Par}$ l'adjectif suivi de l'expression $p o^{1}-t y a^{3}$, a en mourir.

$\left(P_{0}{ }^{1}=\right.$ verbe auxiliaire, arec sens de mourir, etc. $-T y a^{3}=$ marque du futur.) Ex.:

$t c h a^{2} p o^{1}-t y a^{3}$, très-bon: $t s^{3} o^{3} p o^{1}-t y a^{3}$, très-gras.

\section{V. - Noms de Nombre.}

- 47. - En a-hi, les noms de nombre sont:

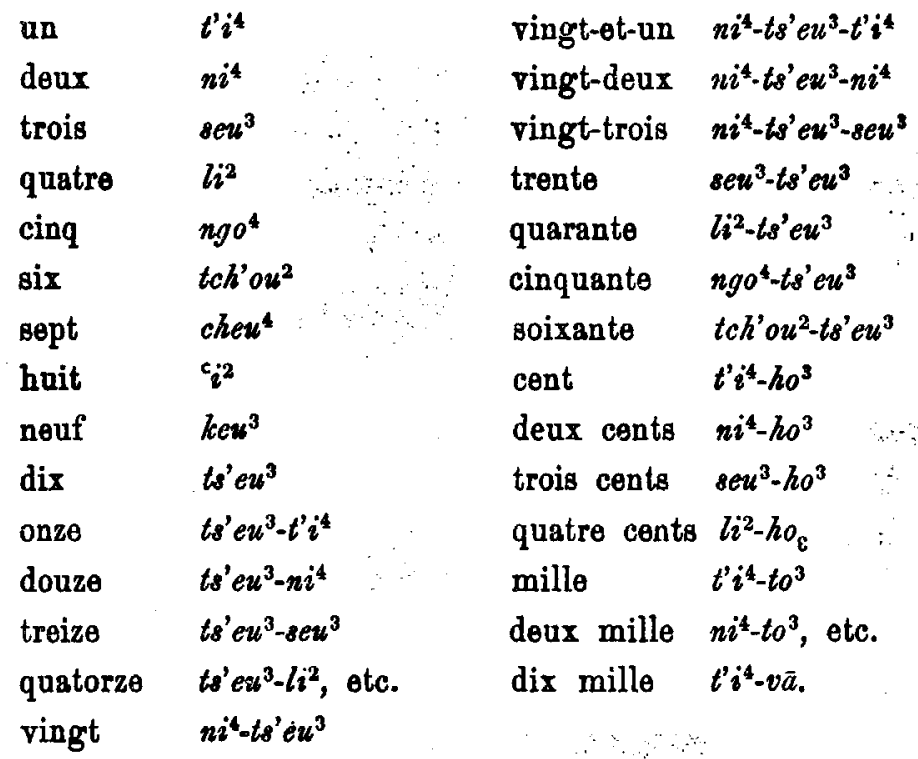

Remarque 1. - On ne dit jamais $h 0^{3}$, cent, tout court; mais un cent, $t^{\prime} i^{4}-h o^{3}$. Même remarque pour $t o^{3}$, mille, et $v \bar{a}$, dizaine de mille.

Rem. 2. - Le mot vãa, dix mille, est le chinois ouan 节.

48. - Les norns de nombre ne sont jamais énoncés seuls: lorsqu'ils ne sont pas suivis d'une numérale spécificative particulière (cf. $\$ 51$ ), on leur ajoute la numérale générale leu ${ }^{4}$, qui correspond 
au chinois ko 個. Un A-hi ne dira donc pas $t^{\prime} i^{4}, t s^{\prime} e u^{3}-k e u^{3}$, cheu

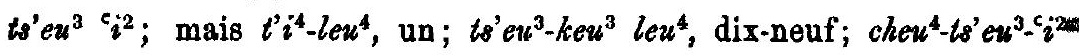
leu, soixante-dix-huit. Ex.:

ts'o ${ }^{3} t^{3} i^{4}-h o^{3} t^{\prime} i^{4}-l e u^{4}$, cent on hommes;

ts'ou ${ }^{3} n i^{4}-h o^{3} s^{3} u^{3} \cdot t s^{\prime} e u^{3}-l e u^{4}$, deux cent trente hommes.

Rem. - Il va de soi que lorsque le substantif auquel est joint le nom de nombre comporte une numérale spécifique (cf. $\$ 51$ ), cette spécificative pent toujours prendre la place de leut. Ex.:

ts'ou $l^{3} l^{2}-t c h ' \hat{e}^{3}$, quatre personnes;

$p^{\prime} o^{3} n g o^{4}$ deu ${ }^{4}$, cinq étoffes.

49. - Lorsque les noms de nombre comportent un nombre rond de centaines, de milliers, ou de dizaines de mille; on ne leur ajoute pas la particule numérale. Ex.:

$t s^{\prime} 0 n^{3} t^{\prime} i^{4}-h o^{3}$, (et non $t s^{\prime} O u^{3} t^{\prime} i^{4}-h o^{3}-l e u^{4}$ ), cent hommes;

$t s^{\prime} 0 u^{3} n i^{4}-t 0^{3}$, deux mille hommes.

ts'ou ${ }^{3}$ seu ${ }^{3}-v \bar{a}$, trente mille hommes.

50. - Le dialecte a-hi possède encore, en dehors de leu ${ }^{4}$, une autre spécificative générale, $m o^{3}$, qui est usité indifféremment pour les personnes et pour les choses. En principe, leub et mo peuvent s'employer avec tous les substantifs; mais il est plus élégant de se servir de la spécificative particulière à chacun. Ex.:

$t s^{\prime} o u^{3} l^{\prime} i^{4}-t c h^{\prime} \hat{e}^{3}$, un homme; mieux que $t s^{\prime} o u^{3} t^{\prime} i^{4}-l e u^{4}$;

$t c h^{3} a^{4}-b o^{3} t^{1} i^{4}-d z e u^{4}$, un palanquin; mieux que $t c h^{1} a^{4}-b o^{3} t^{1} i^{4}-m o^{3}$; $p^{\prime} o^{3} t^{\prime} i^{4}-d e u^{4}$, une toile; mieux que $p^{\prime} o^{3} t^{\prime} i^{4}-m o^{3}$.

51. - Voici la liste des particules spécificatives les plus employées. $b o^{3}$, pour les tas, les monceaux;

$d e^{3}$, pour les huiles;

$d e u^{4}$, pour les étoffes;

$d j 0^{4}$, pour les pagodes;

$d z \hat{e}^{3}$, pour les fagots;

$d z e u^{4}$, pour les chars, les palanquins;

fou ${ }^{1}$, pour les potions;

$h a^{4}$, pour les coups de vent;

hleu, pour les brasses;

$j o^{2}$, pour les affaires, les troupes;

$k^{\prime} a^{2}$, pour les bandes, les blocs, les morceaux; 
$k^{\prime} a^{4}$, pour les fagots;

$k^{\prime} i{ }^{1}$, pour les paroles;

$k^{\prime} i^{2}$, pour les accès de fièvre;

$k i^{3}$, pour les charges des bêtes de somme;

$k 0^{3}$, pour les pipes;

la, pour les paquets, les ballots;

lou

mou ${ }^{2}$, pour les bouchées, les gorgées;

$n y e^{3}$, pour les brèches, les trous, les portes;

$p a^{3}$, poưr les caractères d'écriture;

$p^{\prime} a^{4}$, pour les aiguilles, les lampes;

$p^{\prime} i^{4}$, pour les objets dont deux font la paire (ex.: $k^{\prime} i^{3}-n \bar{o}^{3} t^{\prime} i^{4}-p^{\prime} i^{4}$, un soulier;

$p^{\prime} o^{2} ; p^{\prime} e u^{2}$, pour les fleurs;

$p o^{3}$, pour les averses;

pot, pour les livres;

pyés, pour les parapluies;

sā, pour les bols;

$8 a^{4}$, pour les graines;

$t a^{4}$, pour les assortiments;

$t c h^{\prime} \hat{\varepsilon}^{3}$, pour les personnes;

tché, pour les chaînes de montagnes;

$t$ cho $^{3}$, pour les routes, fil, corde, bracelets, poignées, bandes de terrains, rizières, toile; couteaux ; affaires ;

$t \hat{e}^{3}$, pour les étages;

$t i^{4}$, pour les repas;

to', pour les habits;

to $0^{3}$, pour les pinceaur, les poils, les plumes;

$t^{\prime} o^{3}$, pour les balances, les chaises, les grands bols;

$t^{\prime} 0^{4}$, pour les feuilles;

ts'ế, pour les arbres;

tseu, pour les articles;

ts'en', pour les bottes; les paquets;

tseu ${ }^{3}$; tse $\hat{e}^{3}$, pour los choses doubles;

t's $0^{3}$, pour les bûches;

$v a^{1}$, pour les bordures; 
vou ${ }^{3}$, pour les personnes;

oyé, pour les firdeaux.

52. - Dans un certain nombre de substantifs composés, le second élément n'est autre que la particule spécificative: il reprend ce rôle lorsque le substantif s'accompagne d'uu nom de nombre. Ex.:

$y i^{3}-k o^{3}$, pipe: $y i^{3} t^{3} i^{4}-k o^{3}$, une pipe;

80-pot, livre: so-t' $t^{\prime} i^{4}-p o^{4}$, un livre;

$s e u^{3}-t s^{\prime} \grave{e}^{\prime}$ arbre: $s e u^{3} t^{\prime} i^{4}-t s^{\prime} \hat{e}^{3}$, un arbre.

53. - Règle de position. - La spécificative est toujours plaçée après le nơm de nombre, qui suit lui-même le substantif accompagné ou non d'un adjectif qualificatif. Ex.:

$k e u^{1} z o^{4} s e u^{3}-v o u^{3}, a^{1}-m \hat{e}^{3} n i^{4}-t c h^{\prime} e^{3} b e u^{3}$, il a trois fils et denx filles; m. à m. (de) lui fils trois personnes, filles trois personnes avoir; $t s^{\prime} O u^{3}$ ber ${ }^{3} t^{\prime} i^{4}-t c h^{\prime} e^{3}$, un homme riche;

$t^{\prime} o^{3} s c u^{3}-l o u^{4}$, trois taëls.

54. - Toutefois, s'il s'agit de jours, mois ou années, par exceptions le nom de nombre se place avant le substantif et ne s'accompagne d'aucune spécificative. On dira done $n i^{4} n y i^{3}$, deux jours; seu $^{3} h l o^{3}$, trois mois; $l i^{2} k o u^{2}$, quatro ans.

55. - Adjectif numéral ordinal. - En a-hi, pour dire premier et second, on se sert en général des expressions chinoises $t^{3} i^{1} i^{2}$ (第一) premier, et $t i^{1} e u l^{1}$ (第二), second.

Pour trojsième, quatrième etc., on dira: $\operatorname{sen}^{3}-l e u^{4} t^{\prime} e u^{1}, \mathrm{~m}$. à m. trois celui-là; $l^{2}$-leu $t^{\prime} e u^{1}$, quatre celui-là, etc. Une autre tournure également employée est la suivante: $\operatorname{sen}^{3} n y i^{3} t^{\prime} i^{4}-n y i^{3}$, m. à m., trois jours un jour, c'est-à-dire le troisième jour; $l i^{2} n y i^{3} t^{\prime} i^{4}-n y i^{3}$, le quatrième jour, etc.

56. - Monnaies. - Pour désigner la sapèque, les A.hi ont conservé le mot $y i^{4}-m o^{3}$, partie du nom du petit coquillage, cauris, $y i^{4} \cdot m o^{3}-t^{\prime} o^{3}-z 0^{4}$, qui lour servait autrefois de monnaie. Ils diront donc régulièrement:

$$
\begin{aligned}
& y i^{4}-m o^{3} t^{3} i^{4}-l e u^{4} \text {, une sapèque; } \\
& y i^{4}-m o^{3} n i^{4}-l e u^{4} \text {, deux sapèques; } \\
& y i^{4}-m o^{3} k e u^{3}-l e u^{4} \text {, neuf sapèques. }
\end{aligned}
$$

De 10 ¿ 99, ils comptent par $f \hat{e}^{\prime}$, (du chinois fen 分), c'est-àdire par dizaines de sapèques. Ex.: 
$y i^{4}-m 0^{3} t i^{4}-f e^{3}, 10$ sapèques, $\mathrm{m}$. à $\mathrm{m}$. sapèques une dizaine; $y i^{4}-m o^{3} k e u^{3}-f \hat{e}^{3}, 90$ sapèques;

$y i^{4}-m o^{3} k e u^{3}-f \hat{e}^{3} k e u^{3}-l e u^{4}, 99$ sapèques, $\mathrm{m}$. à $\mathrm{m}$. sapèques neuf dizaines neuf.

De 100 a 999 , ils comptent par $t s^{\prime} \hat{e}^{3}$, (du chinois ts'ien 鐵), c'est-ḋ-dire par centaines de sapèques. Ex.:

$y i^{4}-m o^{3} t^{\prime} i^{4}-t s^{\prime} e^{3}, 100$ eapèques, m. à m. sapèques une centaine;

$y i^{4}-m o^{3} n i^{4}-t s^{\prime} \hat{e}^{3}, 200$ sapèques;

$y i^{4}-m o^{3}$ sen $^{3}-t s^{3} e^{3} t^{\prime} i^{4}-f \hat{e}^{3}, 310$ sapèques;

$y i^{4}-m o^{3} \quad k e u^{3}-t s^{3} e^{3} \quad k e u^{3}-f e^{-3} k e u^{3}-l e u^{4}, 999$ sapèques.

A partie de 1000, ils comptent par lou ${ }^{4}$, c'est-à-dire par ligatures do 1.000 sapèques. Ex.:

$y i^{4}-m o^{3} l^{3} i^{4}-l o u^{4}, 1.000$ sapèques; $\mathrm{m}$. à $\mathrm{m}$. sapèques une ligature. $y i^{4}-m o^{3} \cdot t^{\prime} i^{4}-l o u^{4} k e u^{3}-t s^{\prime} \hat{e}^{3} n i^{4}-f e^{3}{ }^{c^{2}} i^{2}-l c u^{4}, 1.928$ sapèques, $\mathrm{m}$. $\mathrm{a} \mathrm{m}$. sapèques une ligature neuf centaines deux dizaines huit.

57. - Pour l'argent, les A-hi se servent également des mots $f \hat{e}^{\prime 3}, t s^{\prime} e^{3}$, et $l o u^{4}$, accompagnant le mot $t^{\prime} o^{3}$, blanc; argent. Ex.:

$t^{\prime} o^{3} t^{\prime} i^{4}-f \hat{e}^{3}$, un fon d'argent;

$t^{\prime} o^{3} t^{\prime} i^{4}-t s^{\prime} \hat{e}^{3}$, un tz'ien d'argent;

$t^{\prime} o^{3} t^{\prime} i^{4}-l o u^{4}$, une once d'argent.

58. - Mesures. - $1^{0}$ En a-hi, les principales mesures de poids ou de capacité sont: la livre, $k i^{4}$; le 10 e de boisseau, $c h e u^{1}$ (chinois chen 升); le boisseau, $t e u^{3}$ (chin. teou 斗); le picul, $t \bar{a}^{2}$ (chin. $\tan$ 石).

$2^{\circ}$ Pour les mesures de longueur, ils se servent des mesures chinoises, à savoir: le pied, tch'eu (ch. tch'e-tse 尺马); et 10 pieds, tchā (chin.tchang 文). Ils mesurent également par brasse $t^{\prime} i^{4} h l e u^{3}$; et par empan, do deux façons, à savoir $t^{\prime} i^{4}-t^{\prime} e^{3}$, équivalant à l'espace compris entre l'extrêmité du pouce et celle du doigt majeur étendus; et $t^{\prime} i^{4}$-tow ${ }^{1}$, équivalant à l'espace compris eutre l'extrêmité du pouce et celle de l'inder étendus.

La règle est la même que pour les monnaies. Ex.:

$h \bar{o}^{4} t^{3} i^{4}-k i^{4}$, une livre de viande;

$h o^{4}-m o u^{3} t^{3} i^{4}-t e u^{3}$, un boisseau de maïs;

$p^{\prime} o^{3} t^{\prime} i^{4}-h l e u^{3}$, une brasse de toile.

59. - Division du temps. - Chez les A-hi, l'année, k'oun', a, du moins actuellement, la même valeur qu'en chinois. ..... 
Les années sont désignées par les animaux du cycle duodénaire. Ces animaux sont les mêmes qu'en chinois, mais on commence le cycle par le tigre et non par le rat. Voici le cycle a-hi :

$\begin{array}{llcc}1^{0} \text { tigre } & l \bar{o} & 7^{0} \text { singe } & n o u^{1} \\ 2^{0} \text { lièvre } & t i^{4}-h l o^{3} & 8^{0} \text { poule } & y e^{4} \\ 3^{0} \text { dragon } & l 0^{4} & 9^{0} \text { chien } & k i^{4} \\ 4^{0} \text { serpent } & c h a^{1} & 10^{0} \text { cochon } & v y e^{2} \\ 5^{0} \text { cheval } & m o^{4} & 11^{0} \text { rat } & h e^{1} \\ 6^{3} \text { brebis } & j o u^{3} & 12^{0} \text { bouf } & n i^{4} .\end{array}$

Rem. - Pour demander l'âge de quelqu'un, on peut dire: $N i^{3} \quad k^{\prime} a-n o^{3} \quad k^{\prime} o u^{2} l o u^{2}$ ?, m. à m. toi combien ans éconler.

Mais les A-hi ignorent en général leur nombre d'années, et demandent plutôt le nom cyclíque de l'année de naissance:

$N i^{3} a^{4}-m i^{1} k^{\prime} o u^{2}$ ?, m. à m. toi quelle année.

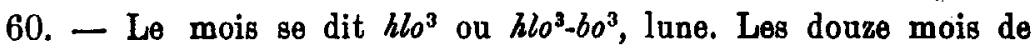
l'année sont parfois désignés par les animaux du cycle duodénaire, comme les jours du mois, du reste. Leurs désignations habituelle sont:

le ler mois $t^{\prime} i^{4}-h l o^{3}$ ou $t c h e u^{4} y i^{2}$ (du chinois tchen iué 正 月);

le 2 e mois $n i^{4}-h l o^{3}$ ou $e u l^{1} y i^{2}$ (du chin. eul iué 二 月);

le $3^{\text {e }}$ mois sen $^{3}-h l o^{3}$;

le $4^{e}$ mois $l i^{2}-h l 0^{3}$;

le $1^{\mathrm{e}}$ mois $k \bar{o} t^{\prime} e u^{1} h l o^{3}, \mathrm{~m}$. à m. tête (?) baisser lune;

le 128 mois $l a^{4}-y i^{2}$ (du chin. la iwé 䐉 月).

61. - Les dix premiers jours du mois prennent en a-hi la particule $d \grave{e}^{3}$, de même qu'en chinois ils prennent la particule $t s^{\prime} o u$ 初. On dira donc: $s e u^{3}-h l^{3} d e^{3} k e u^{3}$, le $9 e$ du $3^{e}$ mois (chin. san iue ts'ou kieou 三月初九); mais on dira $n g o^{4}-h l o^{3} n i^{4}-t s^{\prime} e u^{3}$, le 20 du $5^{\mathrm{e}}$ mois (chin. ou iué eul-che 五.月二十).

62. - Le jour se dit nyi ${ }^{3}$. Voici les divisions du jour chez les A-hi:

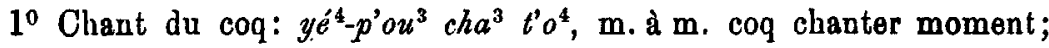

$2^{0}$ Aurore: mou ${ }^{4}-t^{\prime} e w^{2} d j \hat{e}^{3} l^{\prime} o^{4}$, (de) l'aurore moment;

$3^{0}$ Lever du soleil: $l i^{1} \cdot k i^{3}$ dou $-l e^{3} l^{\prime} i^{4}-h \bar{a}$, soleil venir alors;

$4^{0} 9$ heures: $n i^{4} l^{\prime} e u^{2} t^{\prime} o^{4}$, boufs chasser moment;

$5^{0}$ Midi: $l i^{1} \cdot k i^{3} m^{4} u^{4} k o u^{1} d z e u^{4} t^{\prime} o^{4}$, soleil (du) ciel milieu atteindre moment; 
$6^{0} 2$ à 4 heures: $l i^{1}-k i^{3} n g a^{4} t^{3} o^{4}$, soleil grand moment;

$7^{0} 4$ à 5 heures: $n i^{4} d y e^{2} g e u^{3}-l \hat{e}^{3} t^{\prime} o^{4}$, bœufo conduire revenir moment;

$8^{0} 5$ à 6 houres: meu ${ }^{1}-t e u$ ts $\hat{B}^{3} t^{\prime} o^{4}$, feu allumer moment;

$9^{0} 6$ heures: $l i^{1}-k i^{3} t^{\prime} e u^{1} t^{3} o^{4}$, soleil tomber moment;

$10^{0}$ Soir: gou $u^{3}-y i^{2} t^{3} o^{4} k o u a^{3}$, se coucher moment arriver;

$11^{9}$ Nuit: $s e u^{3}-v o u^{3} k o u a^{3}$, nuit arriver;

$12^{0}$ Minuit: $s e u^{3} t^{\prime} o^{3}$, nuit milieu.

Le ler repas, $\left\{\begin{array}{l}n o^{2}-h i^{3} t s o^{3} d z 0^{4}, \mathrm{~m} . \text { a } \mathrm{m} \text {. (du) matin riz manger; } \\ k^{3} i^{4} d z 0^{4}, \mathrm{~m} \text {. a m. repas du matin manger; }\end{array}\right.$

Le repas de midi, $t 80^{3}-d y e^{4} t 8 o^{3} d z 0^{4}$, (de) midi riz manger;

Le repas du soir, $\left\{\begin{array}{l}m e u^{4}-t s^{\prime} i^{2} t s o^{3} d z 0^{4} ; \mathrm{m} \text {. ̀̀ m. (du) soir riz manger; } \\ t c h^{\prime} e u^{3} d z 0^{4}, \text { m. ̊̀ m. repas du soir manger. }\end{array}\right.$

\section{VI. - Pronoms personnels.}

63. - En a-hi, les pronoms personnels sout:

\section{Singulier.}

Première personne $g o^{3} \quad \mathrm{je}$, moi

Deuxième personne $n i^{3} \quad$ tu, toi

Troisième personne $k e u^{1}$ il, lui, elle $k e u^{1}-h i^{4} \quad$ ils, eux, elles.

Remarque 1. - $H i^{4}$, marque du pluriel, est souvent remplaçé par $v i^{3}$. (Ex.: $k e u^{1}-v i^{3}$, eux).

Remarque 2. - En lo.lo, il n'y a pas, à proprement parler, de termes particuliers honorifiques. Aux personnes âgées on donne le nom de $a^{1}$-peu $m^{\prime 4}$, vieillard; ou de $a^{1}-p^{\prime} i^{4}-m^{3}$, vieille-femme. En a-bi, aux personnes qu'on veut honorer, on dit $n a^{1}-v i^{3}$, vous; au lieu de $n i^{3}$, tu. Mais c'est assez rare.

64. - Moi-même peut se dire $g 0^{3}-g 0^{3}$, m. à m. moi moi; soit $g o^{3} \quad t^{\prime} i^{4}-m o^{3}$, moi une personne; soit $g o^{3} t^{1} i^{4}-t c h^{3} e^{3}-z o^{4}$, moi une personne unique. On dira pareillement: $n i^{3}-n i^{3}$, ou $n i^{3} t^{1} i^{4}-m 0^{3}$, ou $n i^{3}$ $t^{\prime} i^{4}-t c h^{\prime} \hat{e}^{3}-z o^{4}$, toi-même; $k e u^{1}-k e u^{1}$, ou $k e u^{1} t^{\prime} i^{4}-m o^{3}$, ou $k e u^{1} t^{\prime} i^{4}-t c h^{\prime} \hat{e}^{3}-$ $z \theta^{4}$, Ini-même.

Rem. - Go $o^{3} t^{1} i^{4}-m o^{3} ; g o^{3} t^{3} i^{4}-t c h e^{3}-z o^{4}$; peuvent signifier aussi moi seul. 
65. - Soi-même se dit $a^{4}-m a^{3}$. En se servant de cette forme jointe au pronom personnel, on peut dire encore pour moi-même, toi-même, lui-même: $g o^{3} a^{4}-m a^{3}, n b^{3} \quad a^{4}-m a^{3}, k e u^{1} a^{4}-m a^{3}$.

Rem. - L'expression $a^{4}-m a^{3}$ a encore le sens de propre, personnel. Ex.:

$A^{4}-m a^{3} m o^{4}$, (mon) cheval propre, m. à m. (de) soi-même (le) cheval.

66. - Partois aussi, mais très-rarement, on emploie l'expression chinoise tsex ${ }^{4}-k i^{3}$ (tse $k i$ 自 已) a la place de $a^{4}-m a^{3}$, et l'on dit: $g 0^{3}$ tseut $-k i^{3}, n i^{3}$ tseu $-k i^{3}, k e u^{1} t s e u^{4}-k i^{3}$.

\section{VII. - Adjectifs ot pronoms possessifs.}

67. - Les adjectifs et pronoms possessifs ne sont antre chose que les pronoms personnels mis au génitif, soit simplement par leur position dans la phrase, soit par l'adjonetion de l'une des particules $d y i^{4}$ ou $v i^{3}$. Dans ce second cas nous avons:

$g 0^{3} d y i^{4}$ ou $g 0^{3} v i^{3}$, de moi, mon, le mien;

$n i^{3} d y i^{4}\left(n i^{3} v i^{3}\right.$ ne se dit jamais), de toi, ton, le tien;

$k e u^{1} d y i^{4}$ ou $k e u^{1} v i^{3}$, de lui, son, le sien;

$g 0^{3}-h i^{4} d y i^{4}$ ou $a^{4}-s e u^{1} d y i^{4}$ (ou dit rarement $g 0^{3}-h i^{4} \quad v i^{3}$ ou $a^{4}-s e u^{1}$ $\left.v i^{3}\right)$, de nous, notre, le nôtre;

$n a^{1}-k i^{4} d y i^{4}$ ou $n a^{1} v i^{3}$, de vous, votre, le vôte ;

$k e u^{1}-h i^{4} d y i^{4}$ (rarement $k e u^{1}-h i^{4} v i^{3}$ ), d'eux, leur, le leur.

Exemples: De qui est-ce la fille? =

$A^{1}-m \hat{e}^{3}-z o^{4} a^{4}-s e u^{3} a^{1}-m \hat{e}^{3}-z 0^{4} n g e u^{3} ? \mathrm{~m}$ ، ̀̀ $\mathrm{m}$. fille (de) qui fille être; or $A^{1}-m \hat{e}^{3}-z o^{4} a^{4}-s e i^{3} d y i^{4} n g e u^{3}$ ?, m. à m. fille qui-de être.

C'est la mienne $=G o^{3} a^{1}-m e^{13}-z 0^{4} n g e u^{3}, \mathrm{~m}$. d̀ m. (de) moi fille être; ou $G o^{3} \lambda_{y i i^{4}} n g e u^{3}, \mathrm{~m}$. à m. moi-de être.

68. - Lorsqu'il s'agit d'un pronom poseessif, c'est-à-dire lorsque le substantif n'est pas exprimé, l'emploi de la particule dyi $i^{4}$ ou $v i^{3}$ signe du génitif, est de rigueur. Mais, devant un substantif, on emploie presque toujours le pronom personnel seul. Ex.:

$G o^{3}$ dyit ngen ${ }^{3}, c^{\prime}$ est le mien;

$M i^{1} \quad k i^{1} l^{1} i^{4}-j o^{2} n g 0^{3} \quad b a^{4} d y i^{4} n g e u^{3}$, cotte bande de torre est celle de mon père, $\mathrm{m}$. à $\mathrm{m}$. terre cette une bande moi père-de être. 
$G o^{3} i^{1}-b a^{4}$, ou $g 0^{3} b a^{4}$, mon père;

$n i^{3} i^{1}-m o^{3}$, ou $n i^{3} m o^{3}$, ta mère;

keu' $z 0^{4}$, son fils.

\section{VIII. - Adjoctifs et Pronoms démonstratifs.}

69. - Les adjectifs démonstratifs, en a-hi, sont: $k i^{1}$ (ou $i^{1}$ ou $e^{1}$ ), ce, cet, cette, celui-ci, et $k i^{1}-t^{\prime} e u^{1}$, ce ... ci, cet ... ci, cette ... ci, celui-ci, pour les personnes et les choses plus rapprochées; $v a^{3}$ (on $\left.k e u^{3}\right)$, ce, cet, cette, celui-ld, et $v a^{3}-t^{\prime} e u^{1}, c \theta . .$. là, cet... là, cette... là, celui-là, pour les personnes et les choses plus élvignées.

70. - La forme simple $k i^{1}\left(i^{1}, e^{\prime}\right)$ se place avant le substantif. Ex. :

$k i^{1} t s^{\prime} o u^{3}$, cet homme;

$k i^{1} t^{\prime}$ 'ou $^{3}$ beu ${ }^{3}$, cet homme riche.

71. - Cependant, lorsque le substantif est accompagné de sa numérale, $k i^{1}$ peut se placer après le substantif ou l'adjectif qualificatif qui l'accompagne, immédiatement avant le nom de nombre. Ex.: dou $k i^{1} t^{\prime} i^{4}-k^{\prime} i^{1}$, cette parole, m. à m. parole cette une, ou $k i^{1}$ $d o u^{4} t^{\prime} i^{4}-k^{\prime} i^{1}$, cette parole une;

$t s^{\prime} O u^{3} b e u^{3} k i^{1} t^{\prime} i^{4}-m o^{3}$, cet homme riche, m. a m. homme riche ce une personne, ou $k i^{1} t s^{\prime} o u^{3} b e u^{3} t^{\prime} i^{4}-m o^{3}$.

72. - La forme composée $k i^{1}-t^{\prime} e u^{1}$ se place après le substantit ou l'adjectif qui le qualifie. Ex.:

$t^{\prime} o u^{3} k i^{1}-t^{\prime} e u^{1}$, cet homme;

$t^{\prime} o u^{3} b e u^{3} k i^{1}-t^{\prime} e u^{1}$, cet homme richo.

73. - Dans la pratique, $k i^{1}$ est presque toujours employé pour ce, ce... ci; et pour ce, ce... là, on se sert seulement de la particule $t^{\prime} e u^{1}$. Ex.:

$z 0^{4} n \bar{a}^{1} t^{\prime} e u^{1}$, ce jeune fils là, m. à m. fils jeune celui-là.

Rem. - A $t^{3} e u^{1}$ se substitue fréquemment $d e u^{3}$, qui s'emploie d'une manière particulière. Ex.:

$t s^{\prime} o u^{3} d e u^{4}-m o^{3}$, cet homme là.

\section{IX. - Adjectifs indéfinis.}

Nous donnerons les principaux.

74. - Aucun se rend par $t^{3} i^{4}-t c h \hat{e}^{3} \quad l \hat{e}^{3} \quad a^{4}$, lorsqu'il s'agit des 
personnes, et par $t^{\prime} i^{4}-m o^{3} n \hat{e}^{3} a^{4}$, lorsqu'il s'agit des choses. Ces expressions se placent après le substantif. Ex.:

$T_{s^{\prime}}{ }^{\prime} u^{3} t^{\prime} i^{4}-t c h^{\prime} e^{3} l e^{3} a^{4} b e u^{3}$, il n'y a personne, m. à m. homme une personne même ne-pas avoir;

$K i^{1} k k^{\prime} y e^{3} h \hat{e}^{3} t^{\prime} i^{4}-m o^{3} n \hat{e}^{3} a^{4} t c h a^{2}$, il n'y a aucune belle maison dans ce village; $\mathrm{m}$. à $\mathrm{m}$. ce village maison une même ne-pas belle.

Rem. - $T^{\prime} i^{4}-m 0^{3} n \hat{e}^{3} a^{4}$, a aussi le sens de rien, rien du tout, m. à m. un même pas; même pas un. Ex.:

$M o u^{4} s a^{4} \cdot p^{3} o^{4} a^{4}-m i^{1} j o^{3} m o u^{4} m i^{1} g o u^{3} ?-T^{1} i^{4}-m o^{3} n e^{3} a^{4} j o^{3}$. De quoi Dieu s'est-il servi pour créer le ciel et la terre? - Il ne s'est servi de rien du tout; $m$. à m. Dieu quoi prendre ciel terre faire? - Un même ve-pas prendre.

75. - Autre se rend par $v a^{4}-n i^{4}$, qui se place avant le substantif. Ex.: $V a^{4}-n i^{1} t s^{\prime} o u^{3}$, d'autres hommes.

76. - Plusieurs, certains, se rendent par l'emploi d'un nom de nombre indéterminé, plus ou moins élevé selon la quantité faible ou grande des objets à indiquer. Ex.:

$T^{\prime} o u^{3} n i^{4} \quad s e u^{3} t c h \hat{e}^{3} d o u^{1}-l e^{3} h o^{3}$, plusieurs hommes sont venus; m. a $\mathbf{m}$. hommes deux trois personnes venir - marque du parfait. $T^{\prime} i^{4}-h o^{3} M o u^{4}-8 a^{4}-p^{\prime} o^{4} d o u^{4}-n \bar{o}^{1} d o^{3}, k i^{1}{ }^{t^{3}} e^{3} t c h a^{2}-m o^{3} d e^{3} b y e^{3} ; t^{\prime} i^{4}-$ $h o^{3} \quad M o u^{4}-s a^{4} p^{\prime} o^{4} d o u^{4} a^{4} n o^{1}, k i^{1} \quad s \hat{e}^{3} k^{\prime} y e^{2}-m o^{3}$ byér $e^{3}$. Certains (les uns) ayant obei \& Dieu; on les appelle bons anges; certains (les autres) ont désobéi, on les appelle mauvais anges; m. à.m. un cent (de) Dieu paroles écouter - marque du passé -, eux esprits bons - particule dépourvue de sens Cf. $\$ 112$ - dire; un-cent (de) Dieu paroles ne-pas écouter, eux esprits mauvais dire.

77. - Quelques peut se rendre de la même manière; mais on se sert plus communément de l'expression $k^{\prime} \bar{a}-n 0^{3}$, qui, prise comme adverbe interrogatif (cf. $\S 100$ ), signifie combien. Ex.:

$t s^{\prime} o u^{3} k^{\prime} \bar{a}-n o^{3} t c h^{\prime} \hat{e}^{3}$, quelques hommes;

$g 0^{3} \quad k^{\prime} \bar{a}-n o^{3} n y i^{3} g e u^{3}-l \hat{e}^{3}$, je reviendrai dans quelques jours.

Rem. - En ajoutant à $k^{\prime} a-n-n o^{3}$ l'expression $a^{4} s a^{1}$; ne pas savoir, on forme un adjectif indéfini équivalant a nombreux, énormément beaucoup. Ex. :

$t s^{\prime} O u^{3}-k^{\prime} \bar{a}-n o^{3} \cdot t c h^{\prime} \hat{e}^{3} a^{4} s a^{1}$, de nombreux hommes.

78. - Tout, tous se rendent par $f o u^{1}-f o u^{3}, i^{4}-b o^{3}-m o^{3}$. Ex.: 
$G o^{3} f o u^{1}-f o u^{3} n g \hat{o}^{1}$ ou $g 0^{3} i^{4}-b o^{3}-m o^{3} n g \bar{o}^{1}$, je veux tout, m. d̀. m. moi tout roulcir;

$A^{4}-8 e u^{3}-l e u^{4}$ fou $-f o u^{3} n e u^{4}-n e u^{4} g o u^{3} ?$ ou plus simplement;

$a^{4}-8 e u^{3}-l e u^{4} f o u^{1}-f o u^{3} g o u^{3}$ ?, qui a créé toutes choses?

$M i^{1}-n y e^{4}-k^{\prime} a^{3} \quad t s^{\prime} o u^{3}$ fou ${ }^{1}-f o u^{3} k e u^{1} n i^{4}-t c h^{\prime} e^{3} d o u^{1}-l \hat{e}^{3}$. Tous les hommes de la terre ont été engendrés par eux deux, m. à $\mathrm{m}$. (de la) terre hommes tous eux deux-personnes venir.

Rem. - Pour rendre toutes choses, on emploie fort bien aussi l'expression $k a^{4}-m i^{1} n g e u^{3} a^{4} n g e u^{3}, m$. à m. quoi être ne-pas être. Ex.: $A^{4}-s e u^{3}-l e u^{4} k a^{4}-m i^{1} n g e u^{3} a^{4} n g e u^{3} g o u^{3}$ ? Qui a créé toutes choses?

79. - Un, un certain se rendent par $t^{3} i^{4}-m o^{3}, t^{\prime} i^{4}-l e u^{4}$, ou $t^{3} i^{4}$ avec la particule spécificative. Ex.:

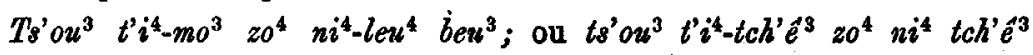
$b e u^{3}$, un homme avait deux fils.

\section{X. - Adjectifs et Pronoms interrogatifs.}

80. - Il y a deux sortes de pronoms interrogatifs: $a^{4}-8 e u^{3}, a^{4}$ seus-leu ou $k a^{4}-s e u^{3}, k a^{4}-s e u^{3}-l e u^{4}$, qui, lequel, pour les personnes; ot $a^{4}-m i^{1}$ on $k a^{4}-m i^{1}{ }^{1}$ ) que, quoi, pour les choses. Ex.:

$Y^{\prime 2}$ sou $^{1} m o u^{4}-k^{\prime} a^{3} d y e^{3} d o u^{1}-k o^{3}, a^{4}-s e u^{3}-l e u^{4} k e u^{1} t c h o^{3}-m a^{3} b y e^{\prime 3}$ ? Après l'ascension, qui a prêché l'Evangile?, m. à m. Jésus ciel monter après, qui (de) lui (la) voie dire?

$N i^{3} a^{4}-m i^{1} m o^{3} ?$ - Que fais-tu?, m. à m. toi quoi faire.

$N i^{3} a^{4}-m i^{1} d z o^{4}$ ? - Que manges-tu?

81. - Au lieu de $a^{3}-s e u^{3}-l e u^{4}$, on peut aussi employer;

$a^{1}-m i^{1} \quad t s^{\prime} o u^{3}$, qui correspond au chinois chen-mo jen 甚麼人, mais cette tournure est assez rare. Ex.:

$A^{4} m i^{1} t g^{\prime} 0 u^{3} k e u w^{1} t c h o^{3}-m a^{3} b y e^{3} ?$ - Qui a prêehé sa doctrine?

1) Cette altermance de $a^{*}$ et de $k a^{4}$, dans $a^{4}-s^{3} u^{3}$ et $k a^{4}-s e u^{3}, a^{4}-m i^{1}$ et $k a^{4}-m i^{1}$, qu'on retronve encore dans l'adverbe interrogatif $a^{4} \cdot m i^{1} \cdot d o^{4}$ on $h a^{4}-m i^{1}-d o^{4}$, pourquoip, exiate ausi pour le mot $a^{4} \cdot b i^{*}$ ou $k a^{4} \cdot b i^{*}$, vêtement. On en peut rapprocher la correspondance $h a^{3}-k^{\prime} \bar{a}$ qui existe pour les quatre adverbes interrogatifs $h a^{*}-z e u^{*}$ ou $k^{\prime} \bar{a}-z \theta u^{*}$, comment?; $h a^{3}-l^{\prime} o^{4}$, on $k^{2} \bar{a}-t^{2} o^{4}$, quand?; $h a^{2}-l e u^{2}$ ou $k^{\prime} a-l e u^{2}$, oul? et $h a^{2} \cdot n o^{3}$ ou $k^{\prime} a$. no", combien? 
82. - $K a^{4}$ de $k a^{4}-s e u^{3}$ peut parfois s'employer seul avec le sens de qui, lequel. Ex.:

$X a^{4} l i^{2} t c h^{\prime} \hat{e}^{3}$ ? quelles sont ces quatre personnes?; m. à m. quelles quatre personnes?

\section{XI. - Verbes.}

83. - En lo-lo, les verbes, comme du reste tous les autres mots, sont invariables. Le nombre et la personne sont indiqués par le sujet exprimé ou soù-entendu.

84. - Des Tomps. - Trois temps seulement, le présent, le passé et le futur, peuvent être exprimés à l'aide de particules spéciales, qui se placent toujours après le verbe et que l'on supprime du reste sourent, lorsque le sens ne les exige pas.

En a-hi, ces trois perticules sont: $c h a^{3}$ pour le présent, $h 0^{3}$ pour lo passé et tya pour le futur. Ex.:

go ${ }^{3}{ }^{80-80 u^{3}}$ cha $^{3}$, j'étudie;

$n i^{3} d z 0^{4} h o^{3}$, tu as mangé;

$k e u^{1} d o u^{1}-l \hat{e}^{3} t y a^{3}$, il viendra.

85. - Ces trois particules s'emploient aussi avec les adjectifs. Ex.: $t^{\prime} \mathrm{Cu}^{3}$ mou $h o^{3}$, homme vieux (qui a vieilli);

tcha $a^{2}$ cha ${ }^{3}$, c'est bon;

hlye's cha $a^{3}$, c'est bouillant;

$h l y e^{3}$ tya $a^{3}$, ça va être chand, bouillant.

86. - En a-hi, en dehors de $h o^{3}$, il existe encore deux particules servant à indiquer le passé: do et no.

$D 0^{3}$ est employé en particulier dans des propositions coordonnés et sert alors à rendre ce qu'on appelle en latin l'ablatif absolu. Ex.: $T^{\prime} o^{3} g o^{3}-l a d o^{3}, m i^{1}-v i^{4}-v i^{4} d o w^{1} d o^{3}$, ayanl ramassé son argent, il s'en alla au loin.

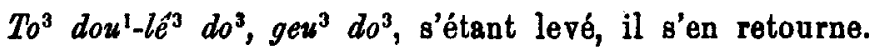

No, est une particule auxiliaire, marque du parfait, avec le sens de avoir déja fait précédemment telle chose.

$N i^{3} d z 0^{4}$ no no?, as-tu déja mangé (de cela)? - Go $d z 0^{4} n o$, j'en ai mangé.

Comme on peut le constater, quand no est employé comme marque 
du passé dans une phrase interrogative, l'interrogation se fait par la répétition de no. Dans certains dialectes, il en est de même pour $h o^{3}$. Ainsi, tandis qu'on $\mathrm{A}-\mathrm{hi}$, on dit:

$n i^{3}{ }^{3} o^{3} d z o^{4} d z o^{4} h o^{3}$, as-tu mangé le riz ?; on dira en On-lou p'ou: $n a^{3} d z o w^{4} h o u^{3} h o u^{3}$ ? - as-tu mangé?

87. - Des Modes. - Voici des phrases montrant la manière dont on peut rendre nos modes en a-hi:

Indicatif présent. - Il étudie, $k e u^{1}{ }^{20-80 u^{3}} c h a^{3}$; il est en train d'étudier.

Imparfait de l'indicatif. - Hier j'étais malade, $g 0^{3} 0^{2}-n y i^{3} \quad n 0^{3}$; moi hier souffrir.

L'an passé, quand il mourut, j'étudiais, $o^{1}-n y i^{3} k^{\prime} o u^{2} k e u^{1} c h e u^{3}$ $t^{3} i^{4}-h \bar{a}, g 0^{3}$ so-sou ${ }^{3}$ cha $a^{3}$ passée-année lui mourir alors, moi étudier en-train.

- Passé. - Il est arrivé, dou $\left.{ }^{1}-l e^{3} a^{3} ;{ }^{1}\right)$ dou ${ }^{1}-1 e^{3} h o^{3}$.

J'ai vu, nyi no; nyi ouo no.

Je n'ai pas vu, $a^{4}$ nyi; nyi $a^{4}$ no.

Plus-que-parfait. - J'avais mangé quand il vint, keu dou ${ }^{1}-l \hat{e}^{3}$ $g 0^{3} \quad t s 0^{3} d z 0^{4} \quad h o^{3}$.

Futur. - Tu mourras, $n i^{3}$ chen ${ }^{3}$ tya ${ }^{3}$.

Je viendrai demain, $g o^{3} a^{4}-d y e^{4} n y i^{3} d o u^{1}-l \hat{e}^{3}$.

Ga ira mal, $a^{4}-d y i^{3} a^{4} t c h a^{2}$; prochainement pas bon.

Futur passé. - J'aurai fini quand tu viendras, $n i^{3} d o w^{1}-l e^{3} g o^{3}$ $k e u^{3} h o^{3}$.

Conditionnel présent. - Tu pourrais certainement, si tu roulais, $n i^{3} p^{\prime} y e^{2} m o^{3} k e u^{3}, t^{1} i^{4}-t i^{1} m o^{3} k e u^{1} a^{3} ; \mathrm{m}$. a $\mathrm{m}$. toi si faire désirer (de bon cceur), sûrement faire être-capable.

Il voudrait bien s'en aller, $k e u^{1} o u o^{1} k^{\prime} o^{4}-k^{3} o^{4}$, lui aller plaise-d̀-Dieu.

Sans cette affaire, je serais libre, $k i^{1}{ }^{2} s u^{4}-t s i^{1} a^{4} n g e u^{3} p^{3} y e^{2}, g o^{3}$ $k y e^{3} m a^{3} ; \mathbf{m}$. ̀̀ $\mathbf{m}$. cette affaire ne-pas être si, moi précisément libre.

Conditionnel passé. - J'aurais fini plus tôt s'il m'avait aidé, $k e u^{1} g o^{3} \quad o u o^{4}-d j o^{3}, k^{\prime} \bar{a} t^{\prime} i^{4}-h \bar{a} \quad t c h \hat{e}^{3} \quad n \hat{e}^{3} \quad g o^{3} \quad g \hat{o}^{3}-k e u^{3} h o^{3}$, m. a m. lui moi aider, combien un-temps vite même moi faire-finir - signe du parfait.

1) Particale finale dépourvae de sens. Cr. 108. 
Impératif. - Mange, $d z 0^{4}$.

Viens, $d o u^{1}-l e^{-3}$.

Qu'il mange, $g 0^{3} \mathrm{keu}^{1} \mathrm{keu} d z o^{4}$; moi lui appeler manger.

$\therefore$ Impératif prohibitif. - Ne dis pas, $t^{\prime} a^{4}$ bye $e^{3}$ (cf. $\S 96$ ).

Optatif. - Plaise à Dieu, $k^{\prime} o^{4}-k^{2} o^{4}$.

Que j'aie des sapèques, $g o^{3} y i^{4}-m o^{3} b e u^{3} k^{3} o^{4}-k^{3} o^{4}-y e^{3} ;$ m. à m. moi sapèques aroir plaise-d̀-Dieu.

Subjonctif présent. - Il demande que tu t'en ailles, $k e u^{1} n i^{3}$ $o u o^{1} m o^{3}$ byé $e^{3} ; \mathrm{m}$. à $\mathrm{m}$. lui toi aller-faire, dire.

Imparfait du subjonctif. - Hier il commanda que je travaillasse, $o^{1}-n y i^{3} \quad k e u^{1} g o^{3} d j o u^{3} b y e^{3} n e u^{4} m o^{3}, \mathrm{~m}$. à $\mathrm{m}$. hien lui moi a dire travail-faire.

Plât a Dieu qu'il fût mort, keư $c h e u^{3} k^{3} o^{4}-k^{3} o^{4}$.

Plus-que-parfait du subjonetif. - S'il m'eût cru, il eût été augsitốt guéri, keu go $o^{3}$ dou $n \bar{o}^{1}, t^{3} i^{4}-l^{3} a^{1}-m o^{3} k y e^{3}$ tcha $a^{2} a^{3} ; \mathrm{m} . \mathrm{a} \mathrm{m}$. lui (de) moi paroles écouter, vite aussitôt bien.

Participe présent. - Regarder en mangeant, $i^{4}-m y e^{1} d z o^{4}, i^{4}-m y e^{1}-$ nyil; $\mathbf{m}$. à $\mathbf{m}$. en même temps manger, en même temps regarder. Prier en marchant, $i^{4}-m y e^{1}$ ouo $, i^{4}-m y e^{1} m o u^{4}-d o u^{4} b y e^{3}, \mathbf{m}$. à $\mathbf{m}$. en même temps marcher, en même temps (da) ciel-paroles dire.

Participe passé. - Faire soi-même, $a^{4}-m a^{3} g o u^{3}$. - Fait par soimême, $a^{4}-m a^{3} g o u^{4}-k i^{4}$.

88. - Verbe passif. - Le passif est formé par la particule tế $\hat{e}^{3}$ Ex. : $d a^{4}$, frapper; $d a^{4}-t e^{3}$, être frappé.

89. - Verbe causal; verbe auxiliaire. - Ces verbes reviennent souvent dans la conversation. Nous avons essayer d'en préciser le sens exact dans le Dictionnaire. Voyez donc aux mots: $d i^{2}=$ pouvoir; possible; $d o^{2}=$ pouvoir, devoir; he $u^{3}=$ pouvoir, facile ; $k e u^{1}=$ pouvoir, habile a...; ts'eu $u^{3}=$ pouvoir; arriver à... - Voyez de plus les mots suivants, jouant le rôle d'auxiliaires: $l \hat{\epsilon}^{3}=$ venir; $m o^{3}=$ faire; $n y e^{3}=$ être; ouo; $r o=$ arriver à; $p o^{1}=$ tomber, renverser; $t o^{3}=$ se lever; etc.

90. - De l'interrogation. - Dans les phrases où elle n'est pas' indiquée par un pronom ou un adverbe interrogatif, l'interrogation s'exprime en a-hi par la répétition du verbe ou de l'adjectif. Ex.:

$N i^{3} s a^{1} s a^{1}$ ? sais-tu?; m. à m. toi saroir eavoir. 
Toha $a^{2}$-tcha $a^{2}$ ? est-ce bon?, m. à m. bon bon.

91. - Dans les adjectifs composés de deux ou plusieurs mots, l'interrogation, au lieu de se faire par la répétition de l'adjectif se fait par la répétition du verbe être. Ex.:

$A^{1} \cdot t^{\prime} o^{3}-m o^{3} n g e u^{3} n g e u^{3}$ ?, est-ce blanc? m. à m. blanc être être.

92. - Toutefois, pour les adjectifs composés terminés en yé (cf. \$34), l'interrogation se fait par la répétition de cette particule, et dans la réponse négative, on peut très-bien n'employer que cette particule sans répéter l'adjectif proprement dit. Prenons, par exemple, $k^{\prime} 0^{1}-y e^{3}$, semblable, le même :

$Y i^{4}-m o^{3} \quad t^{\prime} i^{4}-t s^{\prime} \hat{e}^{3} \quad k^{\prime} e u^{4}, y i^{4}-m o^{3} t^{\prime} i^{4}-l o u^{4} \quad k^{\prime} e u^{4}$, tsou ${ }^{1} \quad l e^{3} t^{\prime} e u^{4}-t^{\prime} e u^{4}$ $k^{\prime} o^{1}-y e^{3}$ ? - $A^{4} y e^{3} ; n e u^{4}-n e u^{4} i^{1}-f a^{3} p^{\prime} o u^{4}-k^{3} y e^{3}$, tsou ${ }^{1} i^{1}-f a^{3} n g a^{4}$. Voler cent sapèques et voler une ligature est-ce la même faute? Non, plus la somme est élevée, plus la faute est grande -; m. à m. sapèques un-cent voler, sapèques une-ligature voler, faute-particule euphon. - semblable semblable? - non semblable; chose davantage chère, faute davantage grande.

93. - De la Négation. - En a-hi, la négation s'exprime par $a^{4}$, qui se place immédiatement avant le verbe ou l'adjectif sur lequel tombe cette négation, lorsque ce verbe ou cet adjectif n'a qu'un seul mot. Ex.:

$G o^{3} a^{4} n g \bar{o}^{-1}$, jo ne veux pas;

$G o^{3} \quad d z o^{4} a^{4} k e u^{1}$, je ne puis manger;

$K i^{1} \quad$ ts' $^{\prime}$ o $^{3} a^{4} t c h a^{2}$, cet homme n'est pas bon.

94. - Dans les verbes composés ${ }^{1}$ ), la négation se place généralement entre les deux mots composant. Ex.:

$b i^{4}-n e u^{1}$, sentir; $b i^{4} a^{4} n e u^{1}$, ne pas sentir.

Remarque. - Je ne connais d'exception à cette règle en a-hi que pour le verbe $d o u^{1}-l e^{3}$, venir, avec lequel la négation se place en tête (a $a^{4} d o u^{1}-l \hat{e}^{3}$, ne pas venir). Mais cette règle de la position de la négation entre les deux éléments d'un verbe composé est loin d'être aussi rigoureuse dans d'autres dialectes lo-lo.

95. - Pas encore se rend en a-hi par $a^{4} \ldots s^{3} e^{3}$ : ces deux particules se placent respectivement avant et après le verbe ou d'adjectif. Ex.:

1) Pour la négation dans les adjectifs composés, cf. $\$ 36$. 
$A^{4} f f a^{3} s e^{3}$, pas encore sec;

$a^{4} d o u^{1}-l e^{3} s e^{3}$, pas encore venu;

$g o^{3} a^{4} d z o^{4} s e^{3}$, je n'ai pas encore mangé;

$g 0^{3} d z 0^{4} a^{4} b e u^{3} s e^{3}$, je n'ai pas mangé encore à satiété.

Rem. - Sé ${ }^{3}$ est un véritable adverbe qui signifie sencores et se place toujours après le verbe ou l'adjectif. Ex.:

$b e u^{3} s e^{3}$, il y en a encore;

$y i^{3} h l y e^{3} s^{3}{ }^{3}$, l'eau est encore chaude.

96. - Il existe en a-hi une autre négation $t^{\prime} a^{4}$ qui, jointe au verbe lui donne un sens prohibitif. Ex.:

$t^{\prime} a^{4} g o u^{3}$, ne fais pas;

$t^{\prime} a^{4} b y e^{3}$, ne dis pas.

Rem. - Souvent à $t^{\prime} a^{4}$, on ajoute encore la particule $k^{\prime} \bar{a}$ pour renforcer le sens prohibitif. Ex.:

$k^{\prime} \bar{a} t^{\prime} a^{4} g o u^{3}$, ne fais pas.

\section{$\therefore \quad$ XII. - Adverbes.}

97. - Les adverbes les plus importants, en dehors de l'adverbe de négation (cf. 93-96), sont les adverbes interrogatifs.

98. - L'adverbe de manière, acomment, est $h a^{3}-z e u^{4}$ ou $k^{\prime} \bar{a}-z e u^{4}$. Ex. :

$A^{4}-s e u^{1} \quad k^{\prime} \bar{a}-z e u^{4} i^{1}-b a^{4} i^{1}-\dot{m} o^{3} o^{1}-m a^{3}-m o^{3} n g o^{-1} ?$ - Comment devonsnous honorer nos père et mère?, $\mathrm{m}$. à $\mathrm{m}$. nous comment père mère servir falloir.

99. - L'adverbe de cause, epourquois, est $a^{4}-m i^{1}-d o^{4}$ ou $k a^{4}-m i^{1}-d o^{4}$. (Dans certains villages on dit $\left.k a^{4}-m i^{1}-d e^{4}\right)$. Ex.:

$M o u^{4} \quad s a^{4}-p^{\prime} o^{4} \quad t^{1} i^{4}-l e u^{4}-z o^{4} \quad n g e u^{3}, a^{4}-m i^{1}-d o^{4} z o^{4}$ beu ${ }^{3} d e^{3} b y e^{3}$ ? Puisque Dieu est un, pourquoi dit-on qu'il a un fils?, m. à m. Dieu unique être, pourquoi fils avoir - partic. euph. - dire.

100. - L'adverbe de quantité, acombiens, est $k^{\prime} \bar{a}-n o^{3}$ ou $h a^{3}-n o^{3}$. Ex.:

$T^{\prime} o u^{3} k^{\prime} \bar{a}-n o^{3} t c h^{\prime} \hat{e}^{3} b^{2} u^{3}$ ? combien y a-t-il d'hommes?

$N i^{3} k^{\prime} a-n o^{3} k k^{\prime} o u^{2} l o u^{2} h o a^{3}$ ? quel âge as-tu? m. \& m. toi combien années écoulées.

101. - L'adverbe de temps, «quands, est $k^{\prime} a \cdot t^{\prime} o^{4}$ ou $h a^{3}-t^{\prime} o^{4}$. Ex.:

$N i^{2} h a^{3} \cdot t^{\prime} o^{4} d o u^{1}-l e^{3}$ ? quand viendras-tu? 
Rem. - Lorsque l'interrogation est plus précise, on peut employer l'expression adverbiale $k a^{4} t^{\prime} i^{4}-h \bar{a}$, en quel temps?

102. - L'adverbe de lieu, soùs est $k^{\prime} \bar{a}-l e u^{1}$ ou $h a^{3}-l e u^{1}$. Ex.:

$N i^{3} h a^{3}-l e u^{1} \quad l i^{3}$ ?, où vas-tu?

Rem. 1. - On peut dire également $h a^{3} l i^{3}$, où aller?, au lieu de $h a^{3}-l e u^{1} l i^{3}$.

Rem. 2. - D'où se rend par $h a^{3}-l e u^{1}-m o u^{4}$, ou $h a^{3}-l e u b^{1}-t c h o^{3}$, ou $h a^{3}-l e u^{1}-m o u^{4}-t c h o^{3}$.

103. - Voici une liste d'autres adverbes fréquemment employés en a-hi.

$k i^{1}-z e n c^{4}$ ainsi, de cette manière $d e^{3}-d \hat{e}^{-3}$ lentement, peu à pea ;

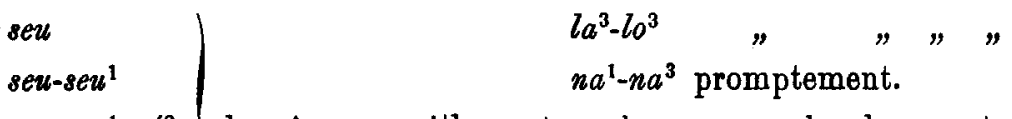

seu-seu ${ }^{1}-y e^{3}$ de mème, pareillement no beaucoup; abondamment.

$k^{\prime} o^{1}$

$k^{\prime} 0^{1}-y e^{3}$

$i^{4}$-myé premièrement; d'abord.

$t^{\prime} e u^{4}-t^{\prime} e u^{4}$ à la fois; ensemble.

$a^{4}-n \grave{e}^{3}$ maintenant

$k i^{1}-t a^{1}$ ici.

$k e u^{3} t^{\prime} i^{4}-h \bar{a}$ en ce temps-là

$i^{4} \cdot t^{\prime}$ ou dessous.

$i^{4}-d 0^{3}$ ensuite, après.

\section{XIII. - Postpositions.}

104. - Les postpositions, qui tiennent en lo-lo la place de nos prépositions, sont peu nombreuses: voici les plus employées en a-hi:

$1^{0} D y i^{4}$ ou $v i^{3}$, «des, marque du génitif. Of. $\$ 29-31$.

$2^{0} D_{j o u}^{3}$, «d marque du datif; ne s'emploie qu' avec le verbe byés, dire. Ex.:

$d z e u^{4}-m o u^{4} k e u^{1}-k i^{4} d j o u^{3} k a^{4}-m i^{1} b y e^{3}$ ?, que leur a dit le mandarin?, m. à. m. mandarin eux à quoi dire.

$3^{0} P y e^{3}$ savecs. Ex.:

$N i^{3} g o^{3} p^{2} e^{3} D o^{4}-8 a^{4}-k o u e^{4} d y e^{3} d y e^{3}$ ? - Viens-tu avec moi à Yun-nan-sen?; m. à m. toi moi avec Yun-nan-sen monter monter, $4^{0} K^{\prime} a^{3}$, esur, dessus, en haut des. Ex.:

$M o u^{4} k^{\prime} a^{3}$, an ciel;

$M i^{4}-n y e^{4} k^{\prime} a^{3}$, sur terre;

Tso $0^{1}-t s e^{3} k^{\prime} a^{3}$, sur la table. 
$5^{0} M o u^{4} ; m o u^{4}-k^{3} a^{3} ; m o u^{4}-t c h o^{3} ; t c h o^{3}$; edes, marquent le point de départ. Ex.:

$G o^{3} \quad D o^{4}-s a^{4}-k o u e^{4}$ mou $u^{4}$ dout $-l \hat{e}^{3}$, ou $g o^{3} \quad D o^{4}-s a^{4}-k o n e^{4}$ mou ${ }^{4}-t c h o^{3}$ $d o u^{1}-l e^{3}$, ou go $D o^{4}-s a^{4}-k o u e^{4} t c h o^{3} d o u^{1}-l \hat{e}^{3}$, je viens de Yun-nan-sen.

$K i^{4}$ tsou $n i^{1}-m o^{3} m o u^{4}-k^{\prime} a^{3} d o u^{1}-l e^{3}$, ce péché vient du ccur.

$6^{0} T c h \bar{a}^{1}$; devant, en présence de. Ex.;

Dze $u^{4}-m o u^{4} t c h a^{1}$, devant le mandarin.

$7^{0} V a^{1}-b o^{4}$, auprès de, à côté de. Ex.:

Dzeu ${ }^{4}-m o u^{4} v a^{1}-b o^{4} k e u$, s'agenouiller à côté du mandarin.

\section{XIV. - Conjonctions.}

105. - Les conjonctions n'existent pour ainsi dire pas en lo-lo. En a-bi, je n'en connais que deux: $n \hat{e}^{3}$, avec le sens rague de set, mêmes, et $k y e^{3}$, c'est-à-dire, cainsi, aussitôtz. On peut du reste toujours les supprimer. Ex.:

$K e u^{1-} h i^{4} t e u^{3}-d 0^{3} b e u^{3}, a^{4}-\operatorname{sen}^{1} n \hat{e}^{3} b e u^{3}$, nous souffrons les mêmes maux qu'eux; $m$. à $m$. eux dommages avoir, nous et (aussi) avoir. $A^{4}-s e u^{1} M o u^{4}-s a^{4}-p^{3} o^{4} d o u^{4} n \bar{o}^{1}, k y e^{3} n y e^{1}-n \bar{o} h o^{4} t s^{\prime} e u^{3}$, en obéissant à Dieu, on peut vaincre le démon; $m$. à $m$. nous (de) Dieu paroles écouter, ainsi démon vaincre pouvoir.

\section{XV. - Particules banales.}

106. - Une des particularités des dialectes lo-lo est le large emploi de particules dépourvues de sens, qui paraissent jouer dans la phrase un rôle purement euphonique.

107. - Nous avons déjà (cf. $\$ 5$ ) des voyelles $a$ et $e ́$ qui s'ajoutent à certains mots terminés en $o$, en $e u$, en $o u$, et qui donnent à cette voyelle la valeur d'une semi-royelle. Ex.:

low', raccommoder, donne toué (lwé1);

ngou $^{1}$, falloir, donne ngoua ${ }^{1}$ (ngwa');

$h o^{3}$, marque du passé, donne $h o a^{3}\left(h w a^{3}\right)$.

108. - La particule $a^{3}$ s'ajoute fréquemment au verbe a la fin d'une phrase et est nettement détachée. Ex.:

$Y e^{\prime 2}-80 u^{1}$ jou hol $0^{3}, i^{1} k^{\prime} o u^{2} \quad l i^{4}-t o^{3} k e u^{3}-h o^{3} \quad t s^{\prime} e u^{3} k^{\prime} o u^{2} l o u^{2} a^{3}$; 
cette année, il y a 1910 que Jésus est né; m. à m. Jésus né (marque passé) cette année un-mille neuf-cent dix ans écoulés.

119. - Cette particule s'ajoute spécialement aux verbes ou aux adjectifs en eu, o, $i$ qu'on emploie seuls dans une réponse affirmative. Ex.:

$H o^{4} t s^{\prime} e u^{3}$, pouvoir vaincre; ou $h o^{4} t s^{\prime} e u^{3} a^{3}$.

$F e u^{3}$, propre; ou $f e u^{3} a^{3}$.

$D i^{2} d i^{2}$ ? - $D i^{2} a^{2}$, est-ce suffisant? - Ca suffit.

$B e u^{3} b e u^{3}$ ? - $B e u^{3} a^{3}, y$ en a-t-il? - Il y en a.

Rem. 1. - Si la réponse est négative, ou n'emploie pas la particule finale $a^{3}$. Ex.;

$A^{4} d i^{2}$, ça ne suffit pas; et non $a^{4} d i^{3} a^{3}$;

$A^{4} b e u^{3}$, il n'y en a pas; et non $a^{4} b e u^{3} a^{3}$.

Rem. 2. - Cette particule peut toujours être supprimée sans nuire au sens ni à l'élégance.

110. - Il existe toute une classe de particules euphoniques qu'on peut toujours supprimer sans nuire à la clarté du sens, mais dont l'emploi donne à la phrase, pour une oreille lo-lo, plus de vivacité et d'élégance. Elles n'ont par elles-mêmes aucun sens, ou perdent du moins, daus cet emploi, celui qu'elles pouvaient avoir à l'origine.

Ces particules, plus ou moins nombreuses suivant les dialectes, sont les suivantes en $\mathrm{a}-\mathrm{hi}: l \hat{e}^{3}, d \hat{e}^{3}, O^{1}$ (prononcé $h o^{1}$ dans certains villages), $y e^{\prime 3}$ et $z 0^{4}$. Cette dernière $z 0^{4}$ avec la première $l \hat{e}^{3}$ sont les seules qui aient, en soi, un sens propre. $Z o^{4}=$ petit, enfant, fils; $l \hat{e}^{3}=$ venir, à. Mais ces sens disparaissent dans leur emploi comme particules euphoniques. Ex.:

$S o^{3} a^{4}-s e u^{1} l e^{3} n \bar{o}^{1} l \hat{e}^{3}, k^{\prime} \bar{a}-z e u^{4} d o u^{4}-k^{\prime} o u^{2} d \grave{e}^{3} n g o a^{1}$ ?, si quelqu'un nous interroge, comment faut-il répondre?; $\mathrm{m}$. à $\mathrm{m}$. autrui nous interroger comment répondre falloir.

$N i^{1}-o u^{2} n i^{1} \cdot v \hat{e}^{3} \quad o^{1} g o u^{3} a^{4} d i^{2} ; t c h a^{2}-t c h a^{2} z o^{4} \quad M_{o u}^{4} \quad s a^{4}-p^{3} o^{4} d o u^{4}$ $z o^{4} o^{1} b y e^{3} n g o^{1} ; n \bar{o}^{3}-t s^{\prime} e^{4}-p^{\prime} o^{4} z o^{4} o^{1} k e u n g o a^{1}$, il ne faut pas faire de pratiques superstitieuses; il faut prier Dieu et appeler le médecin; m. à m. pratiques-superstitieuses faire ne-pas pouvoir; très-bien (de) Dieu paroles dire falloir; médecin appeler falloir.

111. - Lế $\hat{e}^{3}$ 'emploie surtout après le sujet ou à la fin de la phrase. Ex.: $A^{4}-s e u^{3} l \hat{e}^{3}$ mou $m i^{1} g o u^{3}$ ? - Mou $s a^{4}-p^{3} o^{4} l \hat{e}^{3} g o u^{3}$, - qui a créé le ciel et la terre? - C'est Dieu. 
112. - $D \grave{e}^{3}$ s'emploie de préférence avant un verbe simple, surtout avant byés, edires. Ex.:

$K e u^{1} m o^{3} M a^{3}-r i^{4}-a^{3} d e^{3} m \hat{e}^{3}$. - Sa mère s'appelle Marie;

$K a^{4}-m i^{1}-d 0^{4} z o^{4} b e u^{3} d e^{3} b y e^{3}$ ? - Pourquoi dit-on qu'il a un fils?

113. - $O^{1}$ s'emploie surtout avant le verbe gouverné par un autre verbe exprimant le pouvoir, la causalité. Ex.:

$O^{1} \quad g \bar{o}^{3} d i^{2} d i^{2} ? O^{1} g \bar{o}^{3} d i^{2}$. - $O^{1} g \bar{o}^{3} a^{4} d i^{2}$. - Peut-on faire? On peut faire. - On ne peut pas faire.

114. - Z $0^{4}$ peut s'ajouter soit à certains substantifs, soit a la numérale spécifique de certains substantifs, soit à certains adjectifs ou adrerbes. Ex.:

$\left.M o u^{4} s a^{4}-p^{\prime} o^{4} z o^{4}\right)$, pour $M o u^{4} s a^{4} p^{3} o^{4}$, Dieu;

$t^{\prime} i^{4}-t c h^{\prime} \hat{e}^{3} z o^{4}$, une personne, pour $t^{\prime} i^{4}$-tch' $\hat{e}^{3}$;

$t c h a^{2}-t c h a^{2} z o^{4}$, très-bien, pour $t c h a^{2}-t c h a^{2}$.

Rem. - Excepté pour $z 0^{4}$, ces règles de position ne sont pas absolument rigoureuses.

\section{XVI. - Construction de la Phrase.}

115. - L'ordre des termes dans la phrase lo-lo est le suivant: $1^{0}$ sujet; $2^{0}$ complément; $3^{0}$ verbe. Le régime du substantif précéde le substantif et l'adjectif qualificatif le suit. Le compliment indirect se place avant le complément direct. Ex.:

$G o^{3} P e^{\prime}-$ to-lou $d j o^{4}$, j’aime Pierre; m. à m. moi Pierre aimer.

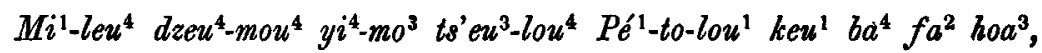
le mandarin de Mi-lé a puni le père de Pierre d'une amende de 10 ligatures; m. à m. (de) Mi-lé (le) mandarin sapèques 10 ligatures Pierre (de) lui père punir-partic. du passé.

116. - Le sujet, - substantif ou pronom - d'une phrase interrogative peut fort bien, pour donner plus d'emphase à l'interrogation, se placer après le complément du verbe. Ex.:

$M o u^{4} m i^{1} a^{4}-s e u^{3}-l e u^{4} g \delta^{3}$ ?, qui a créé le Ciel et la terre? m. à m. Ciel terre qui faire.

1) Cette expression peut assi avoir le sens de ale fils de Dieus. 
$G o^{3}-h i^{4} t s^{\prime} o u^{3} \quad M o u^{4}-s a^{4}-p^{0} o^{4} g \bar{o}^{3} \quad l \hat{e}^{3} \quad n g e u^{3} n g e u^{3} ?$, est-ce Dieu qui nous a créés?, m. à $m$. nous hommes Dieu faire être être.

$T c h a^{3}-z o^{4}, \quad l i^{1}-k i^{3}, \quad h l o^{3}-b o^{3} M o u^{4}-s a^{4}-p^{\prime} o^{4} \quad g o^{-3} n g e u^{3} n g e u^{3}$ ?, est-ce Dieu qui a créé les étoiles, le soleil et la lune?, m. à. m. étoiles, soleil, lune, Dieu faire être être.

Rem. - Ces deux derniers exemples nous montrent la manière de rendre en a-hi nos phrases interrogatives commençant par cest-ces?

117. - Il arrive, mais assez rarement, que le complément indirect soit énoncé après le complément direct.

118. - Les particules indiquant le présent, le passé et le futur se placent après le verbe, à la fin de la phrase: elle en font du reste, en quelque sorte, partie intégrante.

119. - Lorsqu'un verbe, exprimant par exemple le pouvoir, la possibilité, la causalité, etc., gouverne un autre verbe, il se place après ce verbe. Ex.:

by $e^{3} d i^{2}$, on peut dire; m. à m. dire pouroir.

120. - On aura remarqué que nous n'avons pas mentionné les pronoms relatifs: c'est qu'en effet ils n'existent pas en lo-lo. Les propositions relatives se placent purement et simplement après leur antécédent. Ex.:

$M o u^{4}-s a^{4}-p^{\prime} o^{4}, i^{1}-s \hat{e}^{3} f o u^{3}-m o^{3} n g e u^{3}, g e u^{4}-m o^{3} a^{4} b e u^{3}$, Dieu, qui est un pur esprit, n'a pas de corps, m. à m. Dieu, esprit pur être, corps, ne-pas avoir.

De même que pour faciliter les recherches dans le Dictionnaire, je le ferai précéder de textes avec mot-à-mot et traduction littérale, pour mieux faire saisir le génie particulier de ce dialecte A-hi.

Le dialecte A-hi n'est fixé par aucune écriture.

Pour ce qui. concerne le langage journalier, on trouvera dans le Dictionnaire de nombreux exemples, qui en donneront une idée très-exacte.

Pour les sujets de plus longue haleine, je reproduirai d'abord avec l'orthographe adoptée ici la Parabole de l'enfant prodigue, (parue dans le Bulletin de l'Ecole-Française d'Extrême-Orient T. IX, $n^{0} 3$, Juillet-Septembre 1909). 


\section{Parabole de l'enfant prodigue.}

$T s^{\prime} o u^{3} \quad t^{\prime} i^{4}-t c h^{\prime} \hat{e}^{3} \quad z o^{4} \quad n i^{4}-t c h^{\prime} \hat{e}^{3} \quad b e u^{3} . \quad Z o^{4} \quad n \bar{a}^{1} \quad t^{\prime} e u^{1} \quad k e u^{1} \quad b a^{4}$

Homme un fils deux avoir. Fils petit celui-là (de) lui père

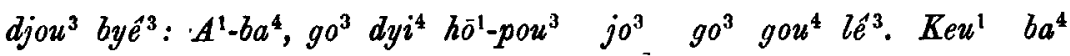
à dire: Père, moi de héritage prendre moi donner. (de) lui père keu ${ }^{1} d y i^{4} h o^{1}-p o u^{3} \quad v i \quad k e u^{1} g e u^{4} h o^{3} . K^{\prime} \bar{a}-n o^{3} \quad n y i^{3} a^{4} \quad l o u^{2}$, lui- de héritage partager lui donner. Combien jours pas écoulés, $z o^{4} \quad n \bar{a}^{1}-m o^{3} k e u^{1} d y i^{4} h o^{1}-p o u^{3}$ fou $-f o u^{3} v o u^{4} h o^{3} . T^{\prime} o^{3} \quad g o^{3}-l a d o^{3}$, fils petit lui de héritage tout vendu. Argent ramassé, $m i^{1}-v i^{4}-v i^{4} d o u^{1} d o^{3}$.

loin sortir.

$K e u^{3}-s \grave{e}^{3}, d o u^{4}-d o u^{1} \quad k e u^{1} \quad t^{\prime} o^{3} \quad t s \bar{o} ; \quad f o u^{1}-f o u^{3} t s \bar{o}$

Là-bas, à-tort à travers (de) lui argent gaspiller; tout gaspiller $k e u^{3} h o^{3} i^{4}-d o^{3}, k e u^{3}-8 e^{3}$ lou ${ }^{1}-n g o u^{4} a^{4} \quad t c h a^{2} ; k e u^{1} \quad d z o^{4} \quad t o^{3} a^{4}$ finir après, là-bas récolte pas bonne; lui manger à ne-pas $b e u^{3}, \quad s o^{3} p^{\prime} y e^{2} v y e^{2} \quad l o u^{1}, \quad o^{4}-p o^{3} n y i^{2} \quad t o^{3}-l \hat{e}^{3}$. Vyé avoir, autrui pour cochons paître, ventre affamé devenir. Cochons $t_{s o^{3}} \quad n g o u^{1} \quad d z 0^{4} \quad n \hat{e}^{3}, \quad s o^{3} \quad j o^{3} \quad k e u^{1} a^{4} \quad t c h \bar{o}$. nourriture désirer manger même, autrui prendre lui ne-pas nourrir. $K e u^{3} t^{1} i^{4}-k \bar{a} k e u^{1} n i^{1}-m o^{3} k i^{1}-z e u^{4} d e u^{4}: \quad g o^{3} \quad b a^{4} \quad t c h \bar{a}^{1}$ Alors lui ccur ainsi songer: (de) moi père en-présence

I. - Un homme avait deux fils. Le plus jeune fils dit à son père: Père, donne moi ma portion d'héritage. Son père ayant fait le partage, lui donna sa part. Après quelques jours, ce jeune fils vendit toute sa part d'héritage; et ayant recueilli son argent, il s'en alla au loin.

Là, il gaspilla malhonnêtement son argent. Ayant tout gaspillé, il arriva qu'en ce pays la récolte fut mauvaise, et il n'eut même pas à manger. Il en fut réduit à faire paître les cochons d'un autre: il ent faim, tellement qu'il désirait manger la nourriture des cochons; mais personne ne lui en donnait à manger. Alors, en lui-même il 
$n e u^{4}-m o^{3}-t s^{\prime} o u^{3} \quad k^{\prime} \bar{a}-n o^{3} \quad t c h o u^{3} ! K e u^{1}-h i^{4} \quad p e^{2}-l e^{2} \quad d z o^{4} \quad t c h a^{2} . G o^{3}$ serviteurs combien être! Eux beaucoup manger bon. Moi $k i^{1}-t a^{1} n y i^{2} \quad p o^{1}-t y a^{3} . G o^{3} g e u^{3}-y i^{3} d o^{3}, g o^{3} \quad b a^{4} \quad d j o u^{3} b y e^{3}: A^{1}-b a^{4}$, ici affamé très. Moi retourner, moi père à dire: Père, $g 0^{3} M o u^{4}-s a^{4}-p^{\prime} o^{4} l \hat{e}^{3} k \hat{\imath} l \hat{e}^{3}, n i^{3} l \hat{e}^{3} k i t \hat{e}^{3} h o a^{3} ; k i^{1}-h o u a^{3} \quad n i^{3}$ moi Dieu offenser, toi offenser; dorénavant (de) toi $z 0^{4} \quad s o \quad a^{4} \quad d i^{2} ; \quad n i^{3} \quad g o^{2} \quad j o^{3} \quad n i^{3} \quad n e u^{4}-m o^{3}-t s^{3} O x^{3} t^{3} i^{4}-$ fils compter ne pouvoir; toi moi prendre (de) toi serviteur un

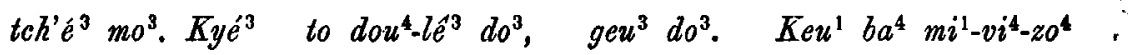

faire. Aussitôt se lever, s'en retourner. Son père au loin $k e u^{1}$ geu ${ }^{3}-l \hat{e}^{3}$ cha $a^{3}$ nyi-ngou ${ }^{3}, c h o u^{2}-m \hat{e}^{3} \quad t o^{3}-l \hat{e}^{3}$.

lui revenir aperçevoir, compatir devenir.

$N a^{1}-n a^{3} t c h \hat{e}^{3} \quad l i^{3}, \quad k e u^{1} \quad l \hat{e}^{3}-r e u^{4}$ tyé-djêt $\hat{e}^{4}, \quad k e u^{1} d j o^{4}-d j o^{4}$

Vite courir aller, (de) lui cou embrasser, lui aimer-aimer

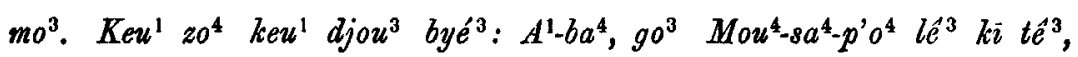
faire. Son fils lui à dire: Père, moi Dieu offenser, $n \hat{\imath}^{3} l \hat{e}^{3} k \bar{\imath} t \hat{e}^{3} h o a^{3}: \quad n i^{3} \quad z o^{4} \quad s \bar{o} \quad a^{4} \quad d \imath^{2} . \quad K e u^{1} \quad b a^{4}$ toi offenser: (de) toi fils compter ne pouvoír. Son père $n e u^{4}-m o^{3}-t s^{\prime} o u^{3}-h i^{4} d j o u^{3}$ bye $e^{3}: N a^{1}-n a^{3} k a^{4}-b i^{4} \quad t s \hat{e}-m o^{3} \quad j o^{3} \quad d o u^{1}-l e^{3}$ serviteurs à dire: Vite habits très-beaux prendre venir

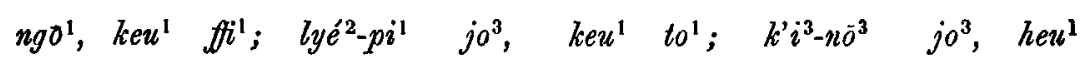
falloir, lui vêtir; anneau prendre, lui enfiler; souliers prendre, lui

pensa: chez mon père nombreux sont les serviteurs; eux ont à manger abondamment; moi ici j'ai faim à en mourir. M'en étant retourné, je veux dire à mon père: Père j'ai offensé et je t'ai offensé. Dorénavant je ne puis compter comme ton fils, mais prends-moi comme ton serviteurs! Et aussitôt s'étant levé, il s'en retourna. Son père l'aperçevant de loin qu'il revenait, fut touché de compassion.

Il courut au-devant de lui, et entourant le cou de son fils, il l'embrassait. Son fils lui dit: «Père, j'ai offensé et Dieu et toi; je ne puis plus compter pour ton filss. Son père dit aux serviteurs: Apportez vite de beaux habits pour l'en revêtir, prenez un anneau et lui enfilez au doigt; prenez des souliers et l'en 


\section{to'. Lo-bou $-z o^{4} \quad t s^{1} o^{3} d e u^{4}-m o^{3} \quad s \hat{e}^{3} \quad d o u^{1}-l \hat{e}^{3}, a^{4}-s e u^{2} \quad h o^{4} \quad d z o^{4}$.} chausser. Veau gras celui-là amener venir, nous tuer manger. $G o^{4} z o^{4} \quad c h e w^{3} h o^{3}, s o^{4} t o^{3}-l e^{3} a^{3} ; k e u^{1} n a^{2} h o^{3}$, chōo $o^{1} h o^{3} a^{3}$. Mon fils mort ressusciter; lui perdu, retrouvé.

$T s o^{3} \quad d z o^{4} \quad c h a^{3} \quad t^{\prime} i^{4}-h a \bar{a}, z o^{4} \quad r a^{4} \quad t^{\prime} e u^{1} m i^{1}-k o u^{1} m o u^{4} g e u^{3}-l e^{3}$,

Riz manger en-train alors, fils grand celui-là champs des revenir, $a^{1}-k^{3} \hat{e}^{-3} \quad k o^{3} \quad d i^{2}-d i^{2} \quad t^{1} i^{4}-h \bar{a}, s a^{4}-n \hat{e}^{1} \quad m o u^{1} \quad n \bar{o}^{1}-d j o^{4}$. Keu ${ }^{1} v i^{3} n e u^{i}-$ maison arriver presque alors, flûte souffer entendre. Lui de $m o^{3}-t s^{\prime} o u^{3} t^{\prime} i^{4}-t c h \hat{e}^{3} \quad k e u$ dou ${ }^{1}-l e^{3} n \bar{o}^{1}$ :

serviteur un appeler venir interroger:

$A^{1}-k^{\prime} e^{3} \quad a^{4}-m i^{1} \quad h i^{4} \quad b e u^{3} ? \quad N e u^{4}-m o^{3}-t s^{3} o u^{3}$ bye $e^{3}: N i^{3} \quad n i^{4}-$

A la maison quelle chose avoir? Serviteur dire: (de) toi frère $k^{3} y e^{3}$ geu $^{3}-l e^{3} h o a^{3} ; n i^{3} b a^{4}$ li $d o^{3}, \quad l o-b o u^{1}-z 0^{4} \quad t s^{\prime} o^{3} d e u^{4}-m o^{3} h o^{4}$ cadet revenu; ton pères'étant réjoui, veau gras celui-là tuer $d z o^{4} . \quad Z o^{4} \quad r a^{4} \quad n i^{1}-h \bar{a} \quad t o^{3}-l \hat{e}^{3}, \quad a^{1}-k^{1} \hat{e}^{3} \quad n \hat{e}^{3} \quad a^{4} \quad g e u^{3}-y i^{3} . K e u^{1}$ manger. Fils grand colère devenir, maison et nepas rentrer. Son $b a^{4} \quad t c h \hat{e}^{3} \quad t^{\prime} e u^{4} \quad l \hat{e}^{3} k e u^{1} l \hat{e}^{3} \quad k e u . \quad K e u^{1} \quad k e u^{1} b a^{4} d o u^{4}-k^{\prime} o u^{2}$ byés père courir sortir lui appeler. Lui (a) son père répondre dire: $G o^{3} \quad k^{\prime} a-n o^{3} \quad k^{\prime} o u^{2} \quad n i^{3} o^{1}-m a^{3}-m o^{3}, \quad n i^{3} \quad d o u^{4} a^{4} \quad n \bar{o}^{1} \quad m o^{3}$, Moi combien années toi servir, (de) toi paroles nepas écouter faire,

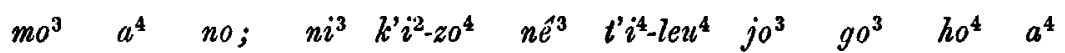
faire ne pas (avoir); toi chevreau même un prendre (à) moi tuer nepas

chaussez. Amenez le veau gras, tuez-le et mangeons. Mon fils était mort, il est ressuscité; il était perdu, le voilà retrouvé! Et tandịs qu'ils festoyaient, le fils aîné revint des champs. Alors qu'il allait arriver à la maison, il entendit le son de la flûte. Appelant un de ses domestiques il lui demanda:

Que se passe-t-il à la maison? Le serviteur répondit: ton frère cadet est revenu; ton père s'en étant réjoui a fait tuer le veau gras pour festoyer. Le fils aîné fort en colère, ne voulut même pas entrer. Son père courut vite l'appeler. Il répondit à son père: je to sers depuis tant d'années! et tes paroles il n'en est pas que je n'ai pas écoutées; or tu n'as même pas tué un chevreau en mon hon- 
$d z 0^{4} \quad m o^{3} . \quad K e u^{1} \quad k e u^{1} d y i^{4} \quad h \bar{o}^{1}-p o u^{3} d o u^{4}-d o u^{1} a^{4}-k a^{3} \quad t s \bar{o} h o^{3}$, manger faire. Lui lui de béritage malhonnêtement gaspiller,

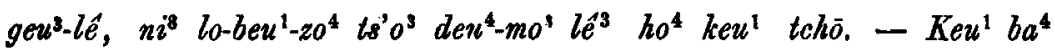
revenir, toi veau gras celui-là tuer lui nourrir. - Son père

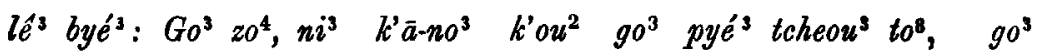

dire: mon fils, toi combien années moi avec être, (de) moi $n e u^{4} n e u^{4} f o u^{1}-f o u^{3} n i^{3} d y i^{4} n g e u^{3} . N i^{3} n i^{4}-k^{\prime} y e^{3}$ cheu $h o^{3} s \bar{o}^{4}-t o^{3} \cdot l \hat{e}^{3}$, affaires tout toi de être. Ton frère-cadet mort ressusciter, $k e u^{1} n a^{2} h o^{3}$, cho $o^{1} h o^{3}, a^{4} \quad l i a^{4} \quad d i^{2} a^{3}$. lui perdu, retrouvé, ne pas se réjouir ne pas pouvoir.

neur. Lui, ayant gaspillé malhonnêtement son avoir, revient; et to lui tues le veau gras. A quoi son père dit: Mon fils: to es avec moi depuis tant d'années, c'est $\nabla$ rai; mais tout ce que j'ai est a toi. Ton frère cadet qui était mort, est ressuscité; il était perdu, le voilà retrouvé. On ne peut pas ne pas se réjouir. 